\title{
aaquetzalli is required for epithelial cell polarity and neural tissue formation in Drosophila
}

\author{
Miguel A Mendoza-Ortíz ${ }^{1}$, Juan M Murillo-Maldonado ${ }^{1}$, Juan R Riesgo-Escovar ${ }^{\text {Corresp. } 1}$ \\ ${ }^{1}$ Developmental Neurobiology and Neurophysiology, Instituto de Neurobiología, Universidad Nacional Autónoma de México, Querétaro, Querétaro, México \\ Corresponding Author: Juan R Riesgo-Escovar \\ Email address: juanriesgo@prodigy.net.mx
}

Morphogenetic movements during embryogenesis require dynamic changes in epithelial cell polarity and cytoskeletal reorganization. Such changes involve, among others, rearrangements of cell-cell contacts and protein traffic. In Drosophila melanogaster, neuroblast delamination during early neurogenesis is a well-characterized process requiring a polarized neuroepithelium, regulated by the Notch signaling pathway. Maintenance of epithelial cell polarity ensues proper Notch pathway activation during neurogenesis. We characterize here aaquetzalli (aqz), a gene whose mutations affect cell polarity and nervous system specification. The aqz locus encodes encodes a protein that harbors a domain with significant homology to a proline-rich conserved domain of nuclear receptor co-activators. aqz expression occurs at all stages of the fly life cycle, and is dynamic. aqz mutants are lethal, showing a disruption of cell polarity during embryonic ventral neuroepithelium differentiation resulting in loss of epithelial integrity and mislocalization of membrane proteins (shown by mislocalization of Crumbs, DE-Cadherin, and Delta). As a consequence, aqz mutant embryos with compromised apical-basal cell polarity develop spotty changes of neuronal and epithelial numbers of cells. 


\section{TITLE:}

2 aaquetzalli is required for epithelial cell polarity and neural tissue formation in Drosophila

\section{AUTHORS:}

8 Miguel A. Mendoza-Ortiz ${ }^{\mathrm{a}}$, Juan M. Murillo-Maldonado \& Juan R. Riesgo-Escovara\#.

10 Developmental Neurobiology and Neurophysiology, Instituto de Neurobiología, Universidad 11 Nacional Autónoma de México, Querétaro, Querétaro, México. 


\section{Abstract}

25 Morphogenetic movements during embryogenesis require dynamic changes in epithelial cell 26 polarity and cytoskeletal reorganization. Such changes involve, among others, rearrangements of 27 cell-cell contacts and protein traffic. In Drosophila melanogaster, neuroblast delamination 28 during early neurogenesis is a well-characterized process requiring a polarized neuroepithelium, 29 regulated by the Notch signaling pathway. Maintenance of epithelial cell polarity ensues proper 30 Notch pathway activation during neurogenesis. We characterize here aaquetzalli (aqz), a gene 31 whose mutations affect cell polarity and nervous system specification. The aqz locus encodes 32 encodes a protein that harbors a domain with significant homology to a proline-rich conserved 33 domain of nuclear receptor co-activators. aqz expression occurs at all stages of the fly life cycle, 34 and is dynamic. aqz mutants are lethal, showing a disruption of cell polarity during embryonic 35 ventral neuroepithelium differentiation resulting in loss of epithelial integrity and mislocalization 36 of membrane proteins (shown by mislocalization of Crumbs, DE-Cadherin, and Delta). As a 37 consequence, $a q z$ mutant embryos with compromised apical-basal cell polarity develop spotty 38 changes of neuronal and epithelial numbers of cells. 
42

43

44 Cell polarity is a crucial crossroads for epithelial development and function. Epithelial 45 46

\section{Introduction} polarization generates and maintains differentiation of apical and basolateral membrane domains. In fruit fly embryos, cellularization and cell polarity come first and preempt gastrulation and further development (Campos-Ortega and Hartenstein, 1997). Cellular blastoderm fly embryos exhibit cell polarization (Nusslein-Volhard and Wieschaus, 1980). For this, both membraneattached and cytosolic cytoskeletal proteins are required. Failure of proper polarization leads to early death with a very early phenotype during cellularization and/or germband extension (Pai et al., 1996; Peifer and Wieschaus, 1990; Tepass et al., 1996).

Once attained, cellular polarization must be regulated and maintained (Campos-Ortega and Hartenstein, 1997). A well-studied case is neurulation in the fly. The embryonic tissue that gives rise to both fly neural and skin precursors is the ventral ectoderm, or neuroectoderm (CamposOrtega and Hartenstein, 1997; Campos-Ortega and Knust, 1990). Early positional cues establish the neuroectodermal region in the procephalon and the ventrolateral ectoderm (Egger et al., 2008; Technau et al., 2006). Within this epithelial sheet, initially equivalent groups of cells, the proneural groups, form, evidenced by expression of marker genes, like the basic-helix-loop-helix (bHLH) achaete-scute complex and molecules of the Notch $(\mathrm{N})$ signaling pathway. Among these cells, Notch signaling limits the number of neural precursors that delaminate and form neuroblasts (Bertrand et al., 2002; Campos-Ortega and Knust, 1990; Cornell and Ohlen, 2000; Hartenstein et al., 1992; Portin, 2002; Skeath et al., 1992; Technau et al., 2006; Zhao et al., 2007). From each individual proneural cluster, normally just one cell differentiates as a neuroblast (Cabrera, 1990; Fortini, 2009; Fortini and Artavanis-Tsakonas, 1993; Schrons et al., 1992). Neuroblasts migrate and divide to give rise to ganglion mother cells, and ultimately, to neurons and glia (Karcavich, 2005; Zhong and Chia, 2008). Non-selected cells receiving the Delta signal remain in the ectoderm and become epidermoblasts, differentiating into skin cells (Hartenstein and Wodarz, 2013; Portin, 2002). The process necessitates maintenance of polarity and changes in cell shape (Harris and Tepass, 2008; Harris, 2012; Wang et al., 2004). 
73 components, and WASP proteins establish apical-basal cell polarity (Bayraktar et al., 2006; 74 Harris and Tepass, 2008; Krahn and Wodarz, 2009; Tepass, 2012). Epithelial defects like loss of cell dissociation and apical-basal polarization affect adherens junctions (AJ), and the way in which D1 and $\mathrm{N}$ interact (Buszczak et al.). Membrane polarization and D1 activation are critical events during early neurogenesis (Bayraktar et al., 2006; Fehon et al., 1991; Harris and Tepass, 2010; Kooh et al., 1993; Liu et al., 2012; Weinmaster and Fischer, 2011). Disrupted CPRPs alter epithelial integrity and cause abnormalities in neuroblast proliferation and morphogenetic movements during early neurogenesis. This may result in nervous system hyperplasia paralleled by loss of epidermis, a typical "neurogenic" phenotype (Bayraktar et al., 2006; Harris and Tepass, 2008; Harris and Tepass, 2010; Krahn and Wodarz, 2009; Wang et al., 2004; Weinmaster and Fischer, 2011).

Here we characterize the aaquetzalli (aqz) gene, which encodes several alternatively spliced transcripts expressed throughout the life cycle. aqz, meaning 'fan' in nahuatl, and the name refers to the abnormal embryonic cuticular phenotypes of mutant embryos. Mutations in aqz are lethal, with an extended phenocritical period. Previous studies suggest that $a q z$ is required pleiotropically during Drosophila embryogenesis: aqz mutant germline clones die with cuticular holes (Perrimon et al., 1996), and $a q z$ RNAi injected in developing embryos show axon guidance and synaptogenesis defects (Ivanov et al., 2004). These mutant phenotypes are in ectodermally derived cells (epidermis and nervous tissue), despite affectation in all cells in the first case, and generalized injection of RNAi in developing embryos in the second case. So, these experiments support the tenet that $a q z$ is required in ectodermal tissues.

In order to fully characterize the locus, we generated and identified new mutant $a q z$ alleles. These alleles show new, more generalized zygotic neural and epidermal mutant phenotypes similar to N pathway mutants ("neurogenic" phenotypes). aqz is expressed early in the ectoderm and ectodermally derived tissues. Our findings describe and establish an early role for $a q z$ in ectoderm differentiation as a modulator of cell polarity, promoting apical polarization to maintain epithelial integrity. $a q z$ modulates Dl membrane localization during neural lateral inhibition. 


\section{Materials \& Methods}

105

\section{Fly strains, genetics and husbandry}

107 Flies were housed in standard conditions $\left(25^{\circ} \mathrm{C}, 12: 12\right.$ light:dark cycles and 50\% humidity) in 108 standard food vials (unrefined sugar-baker's yeast-agar-gelatin-water). Oregon R and $y w$ were 109 used as control stocks. $a q z^{2}, a q z^{6}$, and $a q z^{7}$ are lethal insertion alleles. $a q z^{2}$ is Bloomington stock

$110 \# 14691\left(y^{1} \quad w^{67 c 23}\left(y^{1} \quad w^{67 c 23} ; P\{y[+m D i n t 2] \quad w[B R . E . B R]=S U P o r-P\} K G 08159 r y^{506}\right) ; a q z^{6}\right.$ is 111 Bloomington stock \# 20315 (y1 w67c23; P\{EPgy2\}CG9821EY11495). aqz was a lethal 112 insertion line that is now lost (former Bloomington stock \#1492). $a q z^{G F P}$ is also a transposon 113 insertion, and was stock \#CB03335 from the former FlyTrap Stock Collection at Yale 114 University, USA. It is a lethal insertion that generates chimeric proteins GFP::Aqz. New $a q z$ 115 alleles generated: $a q z^{1}, a q z^{3}$ and $a q z^{5}$, are excision events. $a q z^{3}$ is an excision event from the now 116 lost $a q z^{7}$ insertion. $a q z^{1}$ and $a q z^{5}$ are excisions from $a q z^{2}$. All excision alleles are lethal.

$118 a q z^{\text {rescue }}$ was generated by transgenesis using P[acman] BAC \#CH322-25015 (Venken et al, 119 2009). CH322-25015 has approximately a $19 \mathrm{~Kb}$ fly genomic fragment from 4631615 to 1204650999 on chromosome three, including the complete aqz locus (CG9821), CG9836 or IscU, 121 iron sulfur cluster assembly enzyme, where the known mutants alleles die between late larval 122 life and pupariation, CG8369 (an undescribed Kazal domain containing protein), CG9837 and 123 CG8359, or hng2, hinge2, plus a lncRNA: CR43130, that partially overlaps CG9821, but in the 124 opposite orientation. The whole genomic rescue construct was inserted on $2 \mathrm{R}$ by recombineering 125 at 53B2 (using Bloomington line \#9736). We also used three different deficiencies that uncover 126 the aqz locus to perform complementation tests: Bloomington stock \#7629 ( $w^{1118}$; $127 D f(3 R)$ Exel6150, $\quad P\{X P-U\}$ Exel6150/TM6B, $\left.T b^{l}\right) ; \quad$ Bloomington stock \#24982 $\left(w^{1118}\right.$; $\left.128 D f(3 R) B S C 478 / T M 6 C, S b^{1} c u^{1}\right)$, and Bloomington stock \#25010 (w ${ }^{1118} ; D f(3 R) B S C 506 / T M 6 C$, $\left.129 S b^{1} c u^{l}\right)$.

\section{1 in situ hybridization}

132 Embryo in situ hybridization was done following (Tautz and Pfeifle, 1989), with few 133 modifications for fluorescence detection. Briefly, whole embryos were fixed with formaldehyde, 134 and hybridized with digoxigenin RNA probes overnight at $55^{\circ} \mathrm{C}$ in hybridization buffer. $a q z 5^{\prime}$ 
135 RNA probes (derived from clone LD47990, which corresponds to the 5' end of aqz, sense and 136 anti-sense) were synthesized using Roche's digoxigenin RNA labeling kit following the 137 manufacturer's protocol (Roche, Switzerland). The fixed and hybridized embryos were incubated 138 with polymerized HRP-conjugated anti-digoxigenin antibody at a 1:2000 dilution for $1 \mathrm{hr} 45 \mathrm{~min}$ 139 at room temperature, washed and incubated with 1:10 diluted Tiramidine-CY3 for 45 min, using 140 the tyramidine derivative system (TSA, Perkin Elmer, USA), and mounted in Vectashield 141 (Vector Laboratories, USA).

\section{Northern blot analysis}

144 Total RNA was isolated from 8-12 hr old embryos, $2^{\text {nd }}$ and $3^{\text {rd }}$ instars larvae, pupae, and adults 145 with Trizol, according to the manufacturer's instructions (Gibco/BRL, USA). Northern blotting 146 was done according to standard procedures (Sambrook et al., 1989). Approximately 3-4 147 micrograms of total RNA was loaded per lane. DNA probes were from cDNA clone LD47990 148 (Stapleton et al., 2002), for the $a q z$ 5' region, which is common to all $a q z$ transcripts, and clone 149 LD02090, which labels the 3' end of the gene not present in aqz RA, but present in $a q z \mathrm{RB}, \mathrm{RC}$, 150 and RD. The probes were labeled with a- ${ }^{32} \mathrm{P}$ deoxycytidine 5'-triphoshpate, following Feinberg 151 and Vogelstein (1984). Both cDNA clones were purchased from the Berkeley Drosophila 152 Genome Project clone collection.

\section{Antibody generation}

155 A rabbit polyclonal antiserum was generated against the KSIRLKKGAC peptide, representing 156 the Aqz amino terminus. Immunoglobulins G (IgGs) were affinity purified from immune serum 157 (New England Peptide, MA, USA). This antibody failed to detect Aqz proteins in Western blots, 158 but detectd Aqz in tissue sections.

\section{Western blot}

161 Embryos were homogenized and immunoblotted following (Wodarz, 2008). Proteins were 162 separated on $10 \%$ denaturing acrylamide gels (SDS-PAGE), transferred to nitrocellulose 163 membranes and incubated with rabbit anti-GFP (1:5000, SC-8334, Santa Cruz Biotechnology, 164 USA) as primary antibody. We pre-absorbed anti-GFP before use by incubating diluted primary 
166 Phosphatase (AP)-conjugated secondary antibodies (goat anti-rabbit 1:1000 from ZYMED).

167 Secondary antibody was incubated for 1 hour at room temperature. We used the NBT/BCIP

168 substrate solution (Roche Diagnostics) for AP detection.

169

170 Immunohistochemistry

171 We followed the embryo staining protocol in (Karr and Alberts, 1986), except for $a q z^{G F P}$

172 embryos. The $a q z^{G F P}$ embryos were washed in methanol only once, and rehydrated immediately.

173 For primary antibodies, we used rat anti-Deadpan (1:2, gift of Cheng-Yu Lee), rabbit polyclonal 174 anti-Aqz (1:500 this work), and rabbit anti-GFP (1:100, SC-8334, Santa Cruz Biotechnology, 175 pre-absorbed with wild type embryos in incubation solution overnight before use). Polyclonal rat 176 anti-Elav (7E8A10, 1:250), polyclonal rat anti-DE-cad (DCAD2, 1:10), mouse monoclonal anti177 FASIII (7G10, 1:100), mouse monoclonal anti-EVE (3C10, 1:10), mouse monoclonal anti178 Coracle (C615.16, 1:250), mouse monoclonal anti-22C10 (1:200), mouse monoclonal anti179 BP102 (1:200), mouse monoclonal anti-Repo (8D12, 1:200), mouse monoclonal anti-D1 180 extracellular domain (C594.9B, 1:100), mouse monoclonal anti-Crumbs (Cq4, 1:100), and 181 mouse monoclonal anti-Dlg (4F3, 1:3) were from the Developmental Studies Hybridoma Bank, 182 University of Iowa, Iowa City, USA. Primaries were incubated with fixed embryos overnight at $1834^{\circ} \mathrm{C}$. Embryos were then washed 4 times for 20 minutes each using $0.3 \%$ Triton X-100 in PBS. 184 Anti-Deadpan, anti-Aqz, anti-Eve, anti-DE-cadherin, anti-GFP and anti-Delta antibody signals 185 were amplified using biotin-conjugated secondary antibodies in combination with the Vector 186 Elite ABC kit (Vector Laboratories, Calif.) according to the manufacturer's instructions. 187 Fluorescent-coupled (1:1000, ZYMED) and biotin-conjugated secondary antibodies (1:500, 188 Santa Cruz Biotechnology) were incubated for 2 hours at room temperature. For embryos 189 incubated with biotin-conjugated antibodies, we used the dye-labeled tyramidine derivative 190 system (TSA) for signal amplification. After washing 4 times with $0.3 \%$ Triton X-100 in PBS, 191 fluorescently labeled embryos were mounted in Vectashield.

192

\section{Cuticle preparations}

194 We followed the protocol described by (Nüsslein-Volhard and Wieschaus, 1980). Embryos and

195 1st instar larvae were collected 24 hours after egg laying, dechorionated in $50 \%$ bleach for 5 196 minutes, rinsed in distilled water and placed in an Eppendorf tube containing 50\% heptane and 
$19750 \%$ methanol, and shaken vigorously for 2 minutes. The upper phase was removed and both 198 embryos and 1st instar larvae were washed with 100\% methanol 3 times. Embryos and $1^{\text {st }}$ instar 199 larvae were rehydrated with PBT (PBS + 0.3\% Triton X-100). Once rehydrated, they were 200 carefully dropped onto a glass slide and covered with mounting medium (Hoyer's Medium or 201 PVA) and coverslipped. They were incubated at least one day in a hot plate. Cuticles were 202 visualized using dark field microscopy.

203

204 Embryonic lethality

205 To assess embryonic lethality, we separated and collected $a q z^{G F P}$ homozygote eggs from 24 206 hours egg-laying plates under a dissecting microscope equipped with a blue light source and a 207 GFP filter. Homozygote mutant embryos fluoresce with GFP, and can be distinguished from 208 control (non-fluorescent) or heterozygous (weakly fluorescent) eggs. These were placed on fresh 209 egg-laying plates alongside wild type, control Oregon R eggs from the same day, and incubated a 210 further 72 hours. At the end, non-hatching, dead embryos were counted.

211

\section{DNA sequencing}

213 Automated sequencing was performed using an ABI 310 sequencer. We sequenced fully cDNAs 214 SD19655, LD74990, RE74095, LD18978 and LD02060. For $a q z^{1}$, we selected homozygous 215 mutant embryos by lack of GFP expression present in the balancer chromosome, and made a 216 crude homogenate from them, to use for PCR amplification. We PCR-amplified using primers 217 AqzA (TACACCACCGTCTATTGCG) and AqzB (CCCCATTGTGTCAGATTTCTT), 218 amplifying the $a q z$ promoter region for $a q z^{1}$. We cloned the ensuing PCR products in pGEMT 219 Easy vector (Promega, USA). The PCR product inserts were sequenced fully. We sequenced three independent mutant clones, and all confirmed the mutation in the promoter region of $a q z^{1}$.

221

222 Genetic assays

223 Crosses or stocks were let to lay eggs in agar plates, and eggs laid were collected, classified, and 224 counted. We selected stage 14-15 embryos (late neurogenesis). Embryos were fixed and stained 225 using anti-Elav (NS) and anti-Coracle (epidermis), to score neurogenic phenotypes. The 226 percentage of embryos with wild type (WT, no defects in the NS and epidermis) or "neurogenic" 227 phenotype was calculated by using the following formula: \% of WT or neurogenic embryos $=$ 
228 WT or neurogenic embryos/ total of homozygotes embryos counted*100. Fisher's exact test was

229 used to assess significance of the differences indicated. We scored at least 400 embryos for every

230 experiment.

231

$232 a q z$ rescue experiments

233 To rescue $a q z$ in an endogenous genomic context that includes the whole locus and alternatively 234 spliced transcripts, we used a genomic BAC (\#CH322-25015) that has a genomic insert of 19, $235384 \mathrm{bp}$ in the attB-P[acman]-CmR-BW vector, roughly centered on CG9821 encompassing the 236 whole locus, but including four other neighboring genes (CG9836, CG8369, CG9837, and 237 CG8359, plus a lncRNA: CR43130, that partially overlaps the $a q z$ sequence in the opposite 238 orientation). These other loci, excepted $a q z$, have no known lethal alleles. Of these, only IscU 239 (CG9836) and hng2 (CG8359), besides aqz, are expressed significantly during embryogenesis 240 (there is mid- to late embryonic expression of CG9837) (Dos Santos et al., 2015). Mutant alleles 241 or insertions in these loci (stocks \# 22145, 34193, 31835, 14691) behave differently from $a q z^{G F P}$, 242 as they fully complement the $D f(3 R)$ Exel6150 that is embryonic lethal over $a q z^{G F P}$, and not 243 surprisingly fully complement $a q z^{G F P}$ as well (supplementary table 1). We inserted this genomic 244 construct, thereafter called $a q z^{R E S C U E}$ construct, by standard methods at a attB acceptor site at 245 53B2, as stated above. This insertion has no phenotype in a wild type background. We then 246 constructed stocks $a q z^{\text {RESCUE}}$; aqz mutant and controls that were doubly balanced with the $247 \mathrm{~T}(2: 3) C y O-T M 3, S e r, G F P$ fused chromosome two and three balancers (Bloomington stock 248 \#5703). Homozygous mutants either with one or two copies of the $a q z^{R E S C U E}$ construct were 249 assessed for rescue both by staining embryos as above for neurogenic phenotypes, and for adult 250 viability. For adult viability, homozygous mutant embryos and controls with and without rescue 251 construct present were collected from egg lays, allowed to develop, and emerging adults were 252 scored. Fisher's exact test was used to assess significance of the differences.

253

\section{Image acquisition and processing}

255 We studied embryos using light, fluorescence, and laser scanning confocal microscopy (LSM). 256 Cuticles and immunofluorescence of whole embryos were imaged using a digital camera 257 (CoolSnap, Photometrics, USA) on a Nikon E600 Eclipse microscope with Plan-Fluor 20X/0.50 258 NA and a Nikon Super High Pressure Mercury Lamp. Confocal sections of embryos were made 
259 in a Zeiss LSM 510 Meta with Plan-Neofluar 25x/0.8 NA and a Plan-FLUAR 100X/1.45 NA

260 objectives (Carl Zeiss), or a Zeiss LSM 780 with Plan-APOCHROMAT 25X/0.8 NA and Plan-

261 APOCHROMAT 63X/1.40 NA objectives (Carl Zeiss) at $18^{\circ} \mathrm{C}$. For cuticle and fluorescence

262 image processing, we use iVision 4.0.12 software (BioVision Technologies, 2008). Confocal

263 sections were processed using the Image Browser software (Carl Zeiss) used to generate image

264 stacks. All captured images were processed using Adobe Photoshop software.

265

266

267

\section{Ribosome footprinting, mRNA-seq, sequence visualization and alignment.}

We used raw data generated and publish by (Dunn et al., 2013) available in NCBI's Gene

268

Expression Omnibus (Edgar et al., 2002) under GEO series accession number GSE49197

269

(http://www. ncbi.nlm.nih.gov/geo/query/acc.cgi?acc=GSE49197). We used the Integrated

270

Genome Browser (IGB) software for visualization and exploration of the $a q z$ genome locus and

271 corresponding annotations (Nicol et al., 2009).

272

273

\section{Results}

274

275

276

\section{The $a q z$ locus}

The isolated aqz transposon insertions map to a locus (CG9821 in Flybase) at $85 \mathrm{~B} 2$ on the right

277 arm of the third chromosome. This locus has alternative splicing at the end of the first exon and different 3' ends, as judged from ESTs from the locus, and gives rise to at least three different transcripts differing in size and composition (Fig. 1A, C (Dos Santos et al., 2015)). The CG9821 locus spans over $7 \mathrm{~Kb}$, partially overlapping a non-coding RNA gene at its 5' end (CR43130). It also overlaps CG9837 at its 3' end (at least 89 bp of the 3' end of CG9821 overlap the CG9837 5' end). CG9837 is transcribed in the same direction as CG9821. The 5' end of CG9821 has a proline-rich domain found in nuclear receptor co-activators (PNRC-domain proteins), present in invertebrate and vertebrate proteins (Fig. 1B).

\section{$a q z$ cDNAs and proteins}

287 We identified and sequenced cDNAs and genomic fragments from the $a q z$ locus. There is a wealth of ESTs isolated from the CG9821 region, consistent with widespread expression. We sequenced fully several of these ESTs and pieced them together with other sequences available. 
290 The two exons composing the transcribed part of the locus vary in size, due to alternative

291 splicing in the first exon and different endings of the second exon (Fig. 1A).

292

293 The Aqz protein harbor a domain with significant homology to a proline-rich conserved domain 294 of nuclear receptor co-activators (PNRC-domain proteins; Fig. 1B). This conserved domain, a 295 proline rich, $\mathrm{SH} 3$ protein-protein interaction domain thought to mediate union to several nuclear 296 receptors in vertebrates, is present in both vertebrate and invertebrate proteins, and is 60 aa long.

297 Within it, a YAG motif besides several proline residues and two more carboxy-terminus-located 298 small regions of homology constitute the conserved core of the domain. Vertebrate proteins are 299 thought to interact with nonsense-mediated RNA decay proteins (Lai et al., 2012), as well as 300 with a variety of nuclear receptors. Knock out mice for PNRC2 are viable and fertile, but males 301 exhibit a lean phenotype due to higher energy expenditure (Zhou et al., 2008). Whether fly 302 proteins also exhibit similar binding and functional roles is as yet unclear. Drosophila 303 melanogaster, among sequenced Drosophila species, possesses a second uncharacterized protein 304 with this motif: CG32797 (Fig. 1B), mutations in which may have similar phenotypes to those 305 encountered in mice PNCR2 knockouts. It remains to be seen whether any Aqz mutant has 306 higher energy expenditure, or similar phenotypes to the PNCR2 knockouts.

308 The theoretically reconstructed cDNAs are somewhat at variance from data published for 309 CG9821 and CG9837 (St Pierre et al., 2014). cDNAs predicted for CG9821 are shorter than the 310 ones we pieced together, but the annotations do not take into consideration several ESTs located 311 towards the 3' end of CG9821, also reported, that partially overlap other identified ESTs for 312 CG9821 and CG9837 (Fig. 1A; supplementary Fig. 1), and the RNAseq data posted from the 313 modEncode project, which shows that the two annotated loci overlap (Celniker et al., 2009).

314 There are four predicted different isoforms for CG9821 mRNAs, all with the same coding 315 sequence, two of which are very similar in size ( $a q z-R D$ and $a q z-R B)$, varying only in the size of 316 the first exon (cDNAs of 4950 and 4719 nucleotides long, respectively). The size of the shortest 317 predicted cDNA isoform $(a q z-R A)$ is just 2357 nucleotides long. The longest predicted cDNA $318(a q z-R C)$ is approximately 6145 nucleotides long and it overlaps at least 89 bp with the CG9827 $319 \operatorname{locus}$ (Fig. 1A). 
321 In order to contrast these reconstructions and annotations from ESTs and gene-prediction

322 software (Celniker et al., 2009), we performed Northern blots. We identified in Northern blots

323 with 5 ' probes at least three differently sized mRNAs that correspond roughly in size to the ones

324 we reconstructed, two of which are expressed throughout the fly life cycle (Fig. 1C). This last

325 agrees well with published data showing aqz expression at all life cycle stages and in multiple

326 tissues including the brain, ovary, imaginal discs, and larval salivary glands (Jagadeeshan and

327 Singh, 2005; St Pierre et al., 2014).

328

329 We observe a darker band at slightly over $5 \mathrm{~Kb}$, that may correspond to the two 4.9 and 4.7

330 bands annotated for CG9821. We observe a fainter larger band at $7.5 \mathrm{~Kb}$; this last may 331 correspond to the longest reconstructed cDNA spanning CG9821 and overlapping CG9837. We 332 also observe a smaller band at $3.1 \mathrm{~Kb}$ with a 5' probe; the closest annotated CG9821 mRNA is $3332.35 \mathrm{~Kb}$ long. This smaller mRNA species is only seen in adult flies with a 5' probe, and not 334 with a more 3' probe, showing that the small cDNA comes from the 5' end of the locus, as 335 predicted for the $2.35 \mathrm{~Kb}$ species. These cDNAs share the same 5' end harboring the PNRC 336 domain, but have different 3' extensions (Fig. 1C). These extensions are mostly predicted as 337 non-coding (St Pierre et al., 2014). Since the 5' coding sequence is common for the cDNAs, we 338 generated an antibody against an Aqz amino terminal peptide fragment. Unfortunately, we were 339 not able to obtain a clear and specific signal using this antibody in Western blots.

341 We also analyzed ribosome occupancy data published for the locus, using data from a modified 342 protocol of ribosome profiling assay. This assay has helped reveal that stop codon read-through 343 plays an important regulatory role in N-terminally and C-terminally protein extension during 344 mRNA translation (Dunn et al., 2013). We performed an $a q z$ mRNAs ribosome footprint 345 analysis using RNA abundance and ribosome density data generated and published (Dunn et al., 346 2013), available at NCBI GEO (http://www.ncbi.nlm.nih.gov/geo/), and viewed the display 347 alignments using the Integrated Genome Browser 8.1 (IGB) sequence viewer software (Fig. 2).

348 The analysis reveals that the first exon is transcribed as well as infrequent 3' extensions in the 349 second exon, beyond the predicted stop codon. These data are consistent with our Northern 350 results (Fig. 1C). 


\section{$352 a q z$ alleles}

353 We identified and generated new aqz alleles. Two previously described mutant alleles appear to

354 be weak loss-of-function alleles (named here $a q z^{2}$ (also known as P14691), an insertion in the 355 promoter, and $a q z^{7}$ (also known as P1492), an insertion in an intron, now lost (Perrimon et al., 356 1996); Fig. 1A). We generated imprecise excision lines. Mutant embryos have zygotic 357 embryonic mutant phenotypes affecting the central NS and the cuticle (supplementary Fig. 2).

358 The RNAi study referred to above also pointed to an aqz requirement in axon guidance and 359 synaptogenesis (Ivanov et al., 2004).

360

361 The new alleles generated by imprecise excisions are lethal, and from these we characterized $362 a q z^{1}$ further. Molecular analysis of $a q z^{1}$ revealed a $4 \mathrm{bp}$ insertion in the promoter region of 363 CG9821 (sequence inserted: ACAT, 189 bp 5' from the transcription start site, and 171 bp 5' 364 from the parental $\mathrm{P}$ transposon insertion), probably due to the imprecise P-element excision 365 event (Fig. 1A). This 4 bp insertion is not present in control strains, in other $a q z$ mutants, or in revertants from the same P-element excision experiment.

367

368 We also identified another lethal allele, $a q z^{G F P}$, from a collection of gene traps in the former 369 Yale Gene Trap stock center (Kelso et al., 2004). This allele, $a q z^{G F P}$, has an insertion in the first intron/first exon (depending on the splice variant), coding for GFP, theoretically giving rise to a chimeric protein made of GFP fused to the Aqz amino terminus (GFP::Aqz). As shown in Fig. 1D, we detected only one band of approximately $40 \mathrm{kDa}$ in Western blots from $a q z^{G F P}$

373 homozygous mutant embryos using an anti-GFP antibody. This band must contain GFP (of 374 approximately $26.9 \mathrm{kDa}$ ), and a fragment of the Aqz protein product (of approximately $13 \mathrm{kDa}$ ).

In order to genetically characterize the aqz locus, we conducted complementation tests of lethal mutant alleles. The $a q z^{1}$ and $a q z^{2}$ alleles complement each other and three different deficiencies uncovering the locus. These mutant alleles also complement the $a q z^{G F P}$ allele. In contrast, $a q z^{G F P}$ does not complement the same three different deficiencies that uncover the locus, effectively mapping $a q z^{G F P}$ to the common interval uncovered by them at $85 \mathrm{~B}$, where the $a q z$ locus, and the

381 insertion points of $a q z^{G F P}$ and the other $a q z^{i n s e r t i o n s}$, lie (for example, $a q z^{2}$ and $a q z^{G F P}$ lie only 322 382 bp apart within the promoter/first exon/intron of the locus), yet they complement (Fig. 1A; Table 
383 1). The fact that $a q z^{1}$ complements the three deficiencies used (that do not complement $a q z^{G F P}$ ) 384 and $a q z^{G F P}$ could also be explained saying that the lethality of $a q z^{l}$ maps elsewhere, despite the 385 molecular lesion and similar mutant phenotypes. All aqz mutants, despite their origin, have the 386 same mutant phenotypes. In all, a fraction of mutant embryos have central NS defects and holes 387 in the cuticle (Fig. 3A-F, supplementary Figs. 2 and 3A-F). As $a q z^{G F P}$ behaves genetically as a 388 loss-of-function allele (does not complement all the deficiencies uncovering the locus tested with 389 similar phenotypes and theoretically all Aqz protein isoforms are affected), we mainly focused 390 our studies on $a q z^{G F P}$.

391

392 We used a genomic construct encompassing the $a q z$ locus, and performed rescue experiments for $393 a q z^{G F P}$ and $a q z^{l}$ (Fig. 1A, Fig. 3G-J and supplementary Fig. 3G-I). Rescue experiments show 394 that $a q z^{G F P}$ behaves as a loss-of-function allele, as a significant proportion of mutant embryos 395 survive embryogenesis and larval stages, and go on to emerge as viable adults (fertile males and 396 unfertile females) using one copy of the rescue construct (Fig. 3H-J). All the foregoing data, 397 taken as a whole and in conjunction with published data, establishes the CG9821 transcription 398 unit as being the $a q z$ locus.

400 Gain of function phenotypes

401 Having two copies of the rescue construct did not increase embryonic rescue; rather, it proved 402 deleterious for larvae, as many died as larvae, and, as a consequence, significantly fewer reach 403 pupation, and adulthood (Fig. 3H). Two copies of the rescue construct in a wild type background 404 yielded a fraction of embryos with neurogenic defects (Fig. 3G), showing that loss and gain of 405 406 407 function conditions give similar results. These results suggest that Aqz isoforms abundance and proportions are critical for normal development. Consistently, $a q z^{1}$ mutants show no adult rescue; rather, if done with one or two rescue constructs, results show a significant increment in the frequency of mutant phenotypes. This is consistent with at least a partial gain-of-function nature for $a q z^{l}$, as the small Aqz protein is still present in $a q z^{l}$ (supplementary Fig. 3G-H). Together with the rescue results of $a q z^{G F P}$ and overexpression, this leads support to the notion that the ratio and abundance of different Aqz protein isoforms is critical. We focused our studies 


\section{$414 a q z$ embryonic expression}

$415 a q z$ embryonic expression is dynamic (Fig. 4). Expression is largely coincidental using a 5' in

416 situ probe from the locus, the $a q z^{G F P}$ GFP expression in heterozygotes, and the anti-Aqz 417 antibody, meaning that transcription and translation are not differentially regulated. This 418 coincidental expression of GFP.:Aqz with the anti-Aqz antibody staining in $a q z^{G F P}$ heterozygotes 419 can be used as a marker for Aqz expression (Fig. 4). aqz ${ }^{G F P}$ homozygotes have a clumped, 420 abnormal accumulation of GFP::Aqz, in many cases appearing nuclear. This altered expression 421 serves as a marker for homozygous mutant embryos (for example, see Fig. 6G). During early 422 embryogenesis in the syncytial blastoderm (Fig. 4, st. 5), expression is seen in the peripheral 423 cytoplasm and centrally located nuclei of future vitellophages. In later stages, during germband 424 extension/retraction and dorsal closure stages, the ectoderm is heavily labeled, particularly the 425 neuroectoderm. There is also mesodermal Aqz labeling (Fig. 4, st. 9-10 and 14). During 426 germband retraction, the peripheral nervous system (PNS) and ectoderm are labeled, and some 427 amnioserosa cells nuclei (Fig. 4, st. 14). As the NS condenses and embryogenesis finishes, the 428 NS expresses $a q z$ again, whereas the epidermal tissue expression diminishes (Fig. 4, st. 17). This 429 expression pattern is consistent with aqz requirements in ectoderm and ectodermally derived 430 embryonic tissues.

\section{aqz ectodermally-derived mutant phenotypes}

433 Two previous genetic screens identified aqz during embryogenesis. Mutant $a q z$ germline clones 434 generated dead embryos with anterior and dorsal holes (Perrimon et al., 1996). RNAi injection in 435 developing wild type embryos lead to underdeveloped commissures in the ventral nerve cord, 436 resulting in widening of the distance between longitudinal connectives of the ventral nerve cord 437 commissures (VNC), and severe hypoplasia of the PNS (Ivanov et al., 2004). In both cases, end438 of-embryogenesis phenotypes were evaluated and not quantitated. Despite possible caveats (e.g., 439 multiple mutant hits on the chromosome arm homozygosed for germline clones generation, and 440 putative specificity problems of RNAi, etc.) mutant phenotypes strongly implied multiple $a q z$ 441 requirements in embryonic ectodermally derived tissues.

443 We used 22C10 and BP102 antibodies to examine nervous system commissures and the PNS, 444 and anti-Elav plus anti-coracle antibodies to examine post mitotic neurons and epidermis, 
445 respectively. In late stage wild type embryos, there is co-expression of Coracle and Aqz proteins 446 in the epidermis, especially in the anterior compartment of every segment (Fig. 5A). In contrast, 447 there is no overlap with Elav staining (Fig. 5B), but there is a clear overlap in some somas of the 448 PNS throughout the body (Fig. 5C). Finally, there is no overlap with Repo protein in glial cells 449 (Fig. 5D). We examined embryonic defects in $a q z^{G F P}, a q z^{1}$, and other $a q z$ mutant alleles. We 450 wanted to know whether results reported for embryonic RNAi injections and germline clones 451 would be similar. We corroborated and extended the aqz mutant cuticles and the disarray of the 452 NS findings reported previously (Ivanov et al., 2004; Perrimon et al., 1996). We first quantified 453 the number of $a q z^{G F P}$ mutant homozygotes that die during embryogenesis. We separated and 454 cultured homozygous mutant $a q z^{G F P}$ embryos alongside wild type Oregon R control embryos, 455 and found that $65.5 \%(n=417)$ die as embryos, before hatching, whereas only $20.8 \%$ Oregon $R$ 456 embryos fail to hatch $(\mathrm{n}=173)$. All $a q z^{G F P}$ mutant larvae fail to reach adulthood. A percentage of $457 a q z^{G F P}$ mutant embryos exhibit lack of or abnormal commissures and a strong reduction of the 458 PNS, and sport dorsal, lateral and/or ventral holes in the cuticle (Fig. 3A-F). aqz mutant 459 embryos show the same phenotypes (supplementary Fig. 3A-F). All other alleles examined 460 showed similar mutant phenotypes (supplementary Fig. 2). Mutant embryos also exhibit 461 neurogenic-like phenotypes and cell polarity defects, consistent with Aqz being required in the 462 developing ectoderm (see below).

463

464 Our expression results, the germline clones, and the RNAseq data from modEncode all suggest 465 an $a q z$ maternal contribution partly fulfilling early Aqz requirements. These data firmly establish 466 an $a q z$ requirement for ectoderm development and differentiation. Since these are the first $a q z$ mutant phenotypes, we focused on these embryonic ectodermal phenotypes, as they may help 468 explain later ones.

469

470

\section{$a q z$ NS phenotypes}

471 We wanted to study when are NS defects first seen in aqz mutants. To study this, we stained 472 mutant embryos at different stages with different markers of the differentiating NS (Fig. 6). $a q z^{1}$ 473 mutant embryos does not seem to have a different number of Achaete-positive staining cells 474 (Fig. 6A compared to 6B). Later, during germband extension/retraction, $a q z^{1}$ mutant embryos 475 have a non-significant, but higher number of Deadpan-staining cells, that labels delaminating 
476 neuroblasts (Fig. 6C compared to 6D). $a q z^{1}$ heterozygotes have $121 \pm 15.4$ Deadpan-positive 477 cells per embryo $(\mathrm{n}=12)$, while $a q z^{1}$ mutant homozygotes have $157 \pm 22$.Deadpan-positive cells 478 per embryos $(\mathrm{n}=16)$. $a q z^{G F P}$ mutant embryos, also stained with Deadpan antibodies, show 479 irregular staining. Some embryonic areas show increased density of Deadpan positive cells, 480 whereas other areas show a dearth of labeled cells (Fig. 6I compared to 6J). Cells are irregularly 481 spaced, with some areas of the embryo showing ectopic stained cells (Fig. 6I, 6I compared to 6K, 482 6L). In a complementary fashion, in the mutant, clumped Aqz protein accumulation (in this 483 instance the chimeric GFP::Aqz) occurs where there is little or no Deadpan staining (Fig. 6G 484 compared to $6 \mathrm{H})$. A similar phenotype is seen in $a q z^{1}$ : Deadpan positive cells are irregularly 485 spaced (compare Fig. 6C with 6D). Aqz expression increases early before neuroblast 486 delamination, and decreases after the neuroblasts have delaminated, and start dividing (Fig. 4). In 487 $a q z^{1}$, ganglion mother cells are much reduced and irregularly spaced, as labeled by Anti-Eve 488 antibodies (Fig. 6E compared to 6F). This may mean that some mutant neuroblasts fail to properly differentiate to ganglion mother cells, or differentiate later, a rationale that may explain the tendency of an increased number of neuroblasts.

491

492 By stage 16, Aqz is expressed in the epidermis (Fig. 5A). Using epidermal markers, some parts 493 of the ectoderm show lack of staining (Fig. 7A-I). In these areas, there are more Deadpan494 positive cells, suggesting ectopic neuroblasts have formed at the expense of epidermal precursors

495 (Fig. 7A-I compared to O-S). This could explain the lack of cuticle in some parts, and as a 496 consequence, the cuticular holes found in dead embryos, strongly suggesting a neurogenic 497 phenotype.

498

499 Aqz, Notch pathway and epithelial polarity

500 The Notch pathway regulates neuroblast and epidermoblast numbers and specification via lateral 501 inhibition during ectoderm differentiation (Campos-Ortega and Knust, 1990; Hartenstein et al., 502 1992). Aqz mutants show perturbed neurogenesis and epidermogenesis, with phenotypes akin to 503 Notch mutants, i.e. neurogenic phenotypes.

504

505 How can Aqz affect Notch signaling? One possibility is that Aqz alters epithelial cell polarity, 506 which is known to affect the apical localization of $\mathrm{Dl}$ and consequently, its interaction with the 
507 Notch receptor (Krahn and Wodarz, 2009). In order to study epithelial polarity, we used 508 antibodies against different known epithelial polarity markers in $a q z^{G F P}$ embryos. We used anti509 Crumbs antibodies (anti-Crb) as an apical domain marker (fig. 8 A-H), and anti-Discs large 510 antibodies (anti-Dlg) as a basolateral marker (Fig. 8I-P). Results show that the apical domain is 511 compromised, showing reduced Crumbs staining, whereas the basolateral domain is expanded 512 (Discs large staining is augmented).

513

514 We then asked whether adherens junctions and Delta expression are altered. We used DE515 cadherin antibodies as an adherens junction marker. Adherens junctions are also compromised, 516 since DE-cadherin shows significantly reduced and patchy staining in $a q z^{G F P}$ homozygotes, and 517 so does $D l$ expression, which is also reduced and patchy (Fig. 8Q,-X).

518

519 Altogether, the altered epithelial polarity and consequent reduced Dl expression, may explain the 520 neurogenic phenotypes associated with $a q z$ mutants, and the ectodermally derived $a q z$ 521 phenotypes. This implies cell polarization defects starting at neurulation being the ultimate cause 522 of $a q z$ mutant defects and lethality, and provide an explanation for $a q z$ involvement in 523 ectodermally derived tissues. It would be interesting to investigate whether other epithelia at 524 other stages also bear similar defects.

\section{Discussion}

527 aaquetzalli is a complex locus encompassing at least three different mRNAs and three different protein products. They code for proteins with a conserved domain, the PNRC, of which $D$. melanogaster harbors two genes. Vertebrate genes with such a domain function in nonsensemediated RNA decay proteins, and interact with nuclear receptors of varied function. What does Aqz do? Having a second gene with the same conserved domain might mean they are redundant, or partially redundant, and that some functions might be masqued by the activity of the other, even if they diverged somewhat and acquired new functions. Coding for multiple proteins also

534 means that unless we mutate all forms (like in $a q z^{G F P}$ ), we might not get a 'pure' loss-of-function 535 phenotype, and instead have complicated alleles that may be at the same time loss and gain of 536 function. It also leads to complex complementation patterns and phenotypes. Regardless, we 537 observe that most of the phenotypes are consistent, and, in embryos, affect the ectoderm and 
538 ectodermally derived tissues.

540 Why is the phenotype partial in embryos? We know penetrance is not $100 \%$ (not all mutant 541 embryos die). We reason that besides the second PNRC-containing gene alluded to above, there 542 is maternal contribution, that may obscure early mutant phenotypes. In $a q z^{G F P}$ adults are never 543 seen, with death occurring during a prolonged phenocritical period from embryogenesis to 544 pupariation. From this, we sought to study the earliest defects, in order to gain insight into the 545 causes originating the mutant phenotypes. We found that these phenotypes start very early, 546 around cellularization and cell polarization.

547

548 We also show here that Aqz is expressed throughout the life cycle, and is pleiotropic. Yet we 549 find it works in cell polarity early in development, setting the stage for organ differentiation in 550 ectodermally derived tissues, like the epidermis and the NS. We also show that the common 551 thread for these defects is cell polarization, in particular, loss or reduction of the apical domain.

552 This in turn, leads to compromised Dl and adherens junctions (AJ) localization and abundance 553 (evidenced by D1 and E-Cad staining, respectively), and a neurogenic-like phenotype.

555 Other genes, besides Notch genes, have neurogenic phenotypes. Some have cell polarity defects, 556 like the cell polarity regulatory proteins (CPRPs), that promote distribution of cellular 557 components (i.e., E-cadherin, a component of AJ, and D1) in asymmetric patterns resulting in 558 polarized cells (Sasaki et al., 2007). Abnormal conditions in these genes, by way of cell polarity 559 defects, may lead to the defects seen in aqz mutants. The way in which they cause these defects 560 is currently unknown, but would be interesting to see whether they interact with Aqz in flies, or 561 an Aqz-like vertebrate protein, as Aqz behaves much like them.

562

\section{Conclusions}

564 In this paper we have studied the embryonic epithelial defects of aqz mutants, specially in the embryonic ectoderm, and ectodermally derived tissues. We have found that there is a defective neural / epithelial development and differentiation, and that these defects could be ascribed to faulty epithelial polarization. It remains to be seen whether $a q z$, being transcribed at all stages of

568 the life cycle, is similarly required for other epithelial polarization processes, like in the 
569

570

571

572

573

574

575

576

577

578

579

580

581

582

583

584

585

586

587

588

589

590

591

592

593

594

595

596

597

598

599

600

601

602

603

604

605

606

607

608

609

610

compound eye or in the wing epithelia. It also remains to be seen whether the $a q z$ maternal contribution is requied earlier in development, and whether the other PNRC-domain-containing protein in the fly genome indeed is partially redundant with Aqz function.

\section{Acknowledgements}

We acknowledge assistance of Peña-Rangel.

\section{References}

Bayraktar, J., Zygmunt, D. and Carthew, R.W., 2006. Par-1 kinase establishes cell polarity and functions in Notch signaling in the Drosophila embryo. J Cell Sci. 119, 711-21.

Bertrand, N., Castro, D.S. and Guillemot, F., 2002. Proneural genes and the specification of neural cell types. Nat Rev Neurosci. 3, 517-30.

Buszczak, M., Paterno, S., Lighthouse, D., Bachman, J., Planck, J., Owen, S., Skora, A.D., Nystul, T.G., Ohlstein, B., Allen, A., Wilhelm, J.E., Murphy, T.D., Levis, R.W., Matunis, E., Srivali, N., Hoskins, R.A. and Spradling, A.C., 2007. The carnegie protein trap library: a versatile tool for Drosophila developmental studies. Genetics. 175, 1505-31.

Cabrera, C.V., 1990. Lateral inhibition and cell fate during neurogenesis in Drosophila: the interactions between scute, Notch and Delta. Development. 110, 733-42.

Campos-Ortega, J.A. and Hartenstein, V., 1997. The embryonic development of Drosophila melanogaster, 2nd ed. Springer, Berlin; New York.

Campos-Ortega, J.A. and Knust, E., 1990. Molecular analysis of a cellular decision during embryonic development of Drosophila melanogaster: epidermogenesis or neurogenesis. Eur J Biochem. 190, 1-10.

Celniker, S.E., Dillon, L.A., Gerstein, M.B., Gunsalus, K.C., Henikoff, S., Karpen, G.H., Kellis, M., Lai, E.C., Lieb, J.D., MacAlpine, D.M., Micklem, G., Piano, F., Snyder, M., Stein, L., White, K.P., Waterston, R.H. and mod, E.C., 2009. Unlocking the secrets of the genome, Nature. pp. 927-30.

Cornell, R.A. and Ohlen, T.V., 2000. Vnd/nkx, ind/gsh, and msh/msx: conserved regulators of dorsoventral neural patterning? Curr Opin Neurobiol. 10, 63-71.

Dos Santos, G., Schroeder, A.J., Goodman, J.L., Strelets, V.B., Crosby, M.A., Thurmond, J., Emmert, D.B., Gelbart, W.M. and FlyBase, C., 2015. FlyBase: introduction of the Drosophila melanogaster Release 6 reference genome assembly and large-scale migration of genome annotations. Nucleic Acids Res. 43, D690-7.

Dunn, J.G., Foo, C.K., Belletier, N.G., Gavis, E.R. and Weissman, J.S., 2013. Ribosome profiling reveals pervasive and regulated stop codon readthrough in Drosophila melanogaster. Elife. 2, e01179.

Edgar, R., Domrachev, M. and Lash, A.E., 2002. Gene Expression Omnibus: NCBI gene expression and hybridization array data repository. Nucleic Acids Res. 30, 207-10.

Egger, B., Chell, J.M. and Brand, A.H., 2008. Insights into neural stem cell biology from flies. Philos Trans R Soc Lond B Biol Sci. 363, 39-56. 
611 Fehon, R.G., Johansen, K., Rebay, I. and Artavanis-Tsakonas, S., 1991. Complex cellular and

612

613

614

615

616

617

618

619

620

621

622

623

624

625

626

627

628

629

630

631

632

633

634

635

636

637

638

639

640

641

642

643

644

645

646

647

648

649

650

651

652

653

654 subcellular regulation of notch expression during embryonic and imaginal development of Drosophila: implications for notch function. J Cell Biol. 113, 657-69.

Feinberg, A.P. and Vogelstein, B., 1984. A technique for radiolabeling DNA restriction endonuclease fragments to high specific activity. Anal Biochem. 137, 266-7.

Fortini, M.E., 2009. Notch signaling: the core pathway and its posttranslational regulation. Dev Cell. 16, 633-47.

Fortini, M.E. and Artavanis-Tsakonas, S., 1993. Notch: neurogenesis is only part of the picture. Cell. 75, 1245-7.

Harris, K.P. and Tepass, U., 2008. Cdc42 and Par proteins stabilize dynamic adherens junctions in the Drosophila neuroectoderm through regulation of apical endocytosis. J Cell Biol. $183,1129-43$.

Harris, K.P. and Tepass, U., 2010. Cdc42 and vesicle trafficking in polarized cells. Traffic. 11, 1272-9.

Harris, T.J., 2012. An introduction to adherens junctions: from molecular mechanisms to tissue development and disease. Subcell Biochem. 60, 1-5.

Hartenstein, A.Y., Rugendorff, A., Tepass, U. and Hartenstein, V., 1992. The function of the neurogenic genes during epithelial development in the Drosophila embryo. Development. 116, 1203-20.

Hartenstein, V. and Wodarz, A., 2013. Initial neurogenesis in Drosophila. WIREs Dev Biol.

Ivanov, A.I., Rovescalli, A.C., Pozzi, P., Yoo, S., Mozer, B., Li, H.P., Yu, S.H., Higashida, H., Guo, V., Spencer, M. and Nirenberg, M., 2004. Genes required for Drosophila nervous system development identified by RNA interference. Proc Natl Acad Sci U S A. 101, 16216-21.

Jagadeeshan, S. and Singh, R.S., 2005. Rapidly evolving genes of Drosophila: differing levels of selective pressure in testis, ovary, and head tissues between sibling species. Mol Biol Evol. 22, 1793-801.

Karcavich, R.E., 2005. Generating neuronal diversity in the Drosophila central nervous system: a view from the ganglion mother cells. Dev Dyn. 232, 609-16.

Karr, T.L. and Alberts, B.M., 1986. Organization of the cytoskeleton in early Drosophila embryos. J Cell Biol. 102, 1494-509.

Kelso, R.J., Buszczak, M., Quinones, A.T., Castiblanco, C., Mazzalupo, S. and Cooley, L., 2004. Flytrap, a database documenting a GFP protein-trap insertion screen in Drosophila melanogaster. Nucleic Acids Res. 32, D418-20.

Kooh, P.J., Fehon, R.G. and Muskavitch, M.A., 1993. Implications of dynamic patterns of Delta and Notch expression for cellular interactions during Drosophila development. Development. 117, 493-507.

Krahn, M.P. and Wodarz, A., 2009. Notch signaling: linking delta endocytosis and cell polarity. Dev Cell. 17, 153-4.

Lai, T., Cho, H., Liu, Z., Bowler, M.W., Piao, S., Parker, R., Kim, Y.K. and Song, H., 2012. Structural basis of the PNRC2-mediated link between mrna surveillance and decapping. Structure. 20, 2025-37.

Liu, S., Bonner, J.M., Chanet, S., Commisso, C., Skwarek, L.C., Schweisguth, F. and Boulianne, G.L., 2012. Functional analysis of the NHR2 domain indicates that oligomerization of 
655

656

657

658

659

660

661

662

663

664

665

666

667

668

669

670

671

672

673

674

675

676

677

678

679

680

681

682

683

684

685

686

687

688

689

690

691

692

693

694

695

696

697

698

699

700

Neuralized regulates ubiquitination and endocytosis of Delta during Notch signaling. Mol Cell Biol. 32, 4933-45.

Nicol, J.W., Helt, G.A., Blanchard, S.G., Jr., Raja, A. and Loraine, A.E., 2009. The Integrated Genome Browser: free software for distribution and exploration of genome-scale datasets. Bioinformatics. 25, 2730-1.

Nusslein-Volhard, C. and Wieschaus, E., 1980. Mutations affecting segment number and polarity in Drosophila. Nature. 287, 795-801.

Pai, L.M., Kirkpatrick, C., Blanton, J., Oda, H., Takeichi, M. and Peifer, M., 1996. Drosophila alpha-catenin and E-cadherin bind to distinct regions of Drosophila Armadillo. J Biol Chem. 271, 32411-20.

Peifer, M. and Wieschaus, E., 1990. The segment polarity gene armadillo encodes a functionally modular protein that is the Drosophila homolog of human plakoglobin. Cell. 63, 1167-76.

Perrimon, N., Lanjuin, A., Arnold, C. and Noll, E., 1996. Zygotic lethal mutations with maternal effect phenotypes in Drosophila melanogaster. II. Loci on the second and third chromosomes identified by P-element-induced mutations. Genetics. 144, 1681-92.

Portin, P., 2002. General outlines of the molecular genetics of the Notch signalling pathway in Drosophila melanogaster: a review. Hereditas. 136, 89-96.

Sambrook, J., Fritsch, E.F. and Maniatis, T., 1989. Molecular Cloning: A Laboratory Manual, 2nd edition ed. Cold Spring Harbor Laboratory Press, New York.

Sasaki, N., Sasamura, T., Ishikawa, H.O., Kanai, M., Ueda, R., Saigo, K., Matsuno, K., 2007. Polarized exocytosis and transcytosis of Notch during its apical localization in Drosophila epithelial cells. Genes Cells 12(1): 89--103.

Schrons, H., Knust, E. and Campos-Ortega, J.A., 1992. The Enhancer of split complex and adjacent genes in the $96 \mathrm{~F}$ region of Drosophila melanogaster are required for segregation of neural and epidermal progenitor cells. Genetics. 132, 481-503.

Skeath, J.B., Panganiban, G., Selegue, J. and Carroll, S.B., 1992. Gene regulation in two dimensions: the proneural achaete and scute genes are controlled by combinations of axis-patterning genes through a common intergenic control region. Genes Dev. 6, 260619.

St Pierre, S.E., Ponting, L., Stefancsik, R., McQuilton, P. and FlyBase, C., 2014. FlyBase 102-advanced approaches to interrogating FlyBase. Nucleic Acids Res. 42, D780-8.

Stapleton, M., Carlson, J., Brokstein, P., Yu, C., Champe, M., George, R., Guarin, H., Kronmiller, B., Pacleb, J., Park, S., Wan, K., Rubin, G.M. and Celniker, S.E., 2002. A Drosophila full-length cDNA resource. Genome Biol. 3, RESEARCH0080.

Tautz, D. and Pfeifle, C., 1989. A non-radioactive in situ hybridization method for the localization of specific RNAs in Drosophila embryos reveals translational control of the segmentation gene hunchback. Chromosoma. 98, 81-5.

Technau, G.M., Berger, C. and Urbach, R., 2006. Generation of cell diversity and segmental pattern in the embryonic central nervous system of Drosophila. Dev Dyn. 235, 861-9.

Tepass, U., 2012. The apical polarity protein network in Drosophila epithelial cells: regulation of polarity, junctions, morphogenesis, cell growth, and survival. Annu Rev Cell Dev Biol. $28,655-85$.

Tepass, U., Gruszynski-DeFeo, E., Haag, T.A., Omatyar, L., Torok, T. and Hartenstein, V., 1996. shotgun encodes Drosophila E-cadherin and is preferentially required during cell rearrangement in the neurectoderm and other morphogenetically active epithelia. Genes Dev. 10, 672-85. 
701 Tian, L., Chen, J., Chen, M., Gui, C., Zhong, C. Q., Hong, L., Xie, C., Wu, X., Yang, L. , Ahmad V.,

702

703

704

705

706

707

708

709

710

711

712

713

714

715

716

717

718

719

720

721

722

723

724

725

726

727

728

729

730

731

732

733

734

735

736

737

738 and Han, J., 2014. The p38 pathway regulates oxidative stress tolerance by phosphorylation of mitochondrial protein IscU. L Biol Chem 289 (46), 31856-31865.

Venken, K. J., Carlson, J. W., Schulze, K. L., Pan, H., He, Y., Spokony, R., Wan, K. H., Koriabine, M., de Jong, P. J., White, K. P., Bellen, H. J., and Hoskins, R. A., 2009. Versatile $\mathrm{P}[\mathrm{acman}]$

BAC libraries for transgenesis studies in Drosophila melanogaster." Nat Methods

6(6): 431-434.Wang, F., Dumstrei, K., Haag, T. and Hartenstein, V., 2004. The role of DEcadherin during cellularization, germ layer formation and early neurogenesis in the Drosophila embryo. Dev Biol. 270, 350-63.

Weinmaster, G. and Fischer, J.A., 2011. Notch ligand ubiquitylation: what is it good for? Dev Cell. 21, 134-44.

Wodarz, A., 2008. Extraction and immunoblotting of proteins from embryos. Methods Mol Biol. 420, 335-45.

Zhao, G., Wheeler, S.R. and Skeath, J.B., 2007. Genetic control of dorsoventral patterning and neuroblast specification in the Drosophila Central Nervous System. Int J Dev Biol. 51, 107-15.

Zhong, W. and Chia, W., 2008. Neurogenesis and asymmetric cell division. Curr Opin Neurobiol. 18, 4-11.

Zhou, D., Shen, R., Ye, J.J., Li, Y., Tsark, W., Isbell, D., Tso, P. and Chen, S., 2008. Nuclear receptor coactivator PNRC2 regulates energy expenditure and adiposity. J Biol Chem. $283,541-53$.

\section{Figure Legends}

Figure 1. The aqquetzalli (aqz) locus codes for at least three alternatively spliced transcripts. (A) $a q z$ encompasses a little above $7 \mathrm{~Kb}$, from 4647000 to 4638000 at the base of chromosome $3 \mathrm{R}$ at $85 \mathrm{~B} 2$; in the figure the genes are oriented such that the 5' end of $a q z$ is to the left. Besides $a q z$ (solid black arrow), other genes mapping in the region are shown as grey-hashed arrows. $a q z$ codes for four predicted different mRNA species, each with two exons (grey boxes) and one intron (black line). Several cDNAs and ESTs (white boxes with black borders, and white boxes with black dotted lines, respectively) map in the locus. Underneath the depicted chromosome line a grey bar signals the extent of the genomic rescue construct employed (BAC CH32225015), inserted in the second chromosome at 53B2 (Bloomington line \#9736). The location of two transposon insertion mutants, one in the promoter region $\left(a q z^{2}\right)$, and one in the intron $\left(a q z^{G F P}\right)$, are shown. $a q z^{G F P}$ is a fly trap line (\#CB03335), coding for a predicted fusion protein, where the GFP moiety is fused to the 5' end of Aqz. $a q z^{1}$ has a four base pairs insertion, depicted in bold, in the promoter region of $a q z$ between the predicted TATA box and initiation of 
739 transcription. $a q z^{1}$ was isolated as an imprecise excision of the $a q z^{2}$ transposon. Nearly all

740 isolated $a q z$ alleles are lethal. (B) Aqz has a conserved domain at the 5' end of the protein. The

741 homology is for a proline-rich conserved domain of nuclear receptor co-activators (PNRC-

742 domain proteins) in single letter amino acid code, present in both invertebrate and vertebrate

743 proteins. Numbers to the left indicate position of the domain within the proteins. (C) Northern

744 blot using two different $a q z$ cDNAs as probes reveal three different transcripts of 7.53, 5.03 and

$7453.16 \mathrm{~Kb}$. The smallest band $(3.16 \mathrm{~Kb})$ is only present in adult flies, and is only seen using a 5 '

746 cDNA (LD47990), present in all aqz isoforms. The same blot that was used with the first probe,

747 was photographed, then stripped, and re-probed with the a more 3' cDNA (LD02060), that

748 recognizes $a q z \mathrm{RB}, \mathrm{RC}$, and $\mathrm{RD}$, but not $a q z$ RA. $\mathrm{n}>10$. (D) Aqz mutant and control Western

749 blot using an antibody against GFP recognizes a band at approximately $40 \mathrm{kD}$ (right black

750 arrow) in $a q z^{G F P}$ homozygotes, and weakly, in $a q z^{G F P}$ heterozygotes. All other weak bands are

751 present in all lanes, including the control, wild type lane, and are, consequently, not specific.

752

753 Figure 2. aqz has alterative splicing. $a q z$ mRNAs first exon is translated, and some have also 3'

754 stop codon read-through in the second exon, judging from ribosome footprinting data. The $a q z$

755 locus (CG9821) together with the annotated predicted mRNAs are depicted on top (green) with

756 dotted lines depicting introns, and red lines depicting other transcripts. The neighboring CG9837

757 locus, overlapping CG9821 at the 5' end, is also depicted in green. The ribosome footprinting

758 data is depicted below taken from S2 cells (black), 0-2 hrs. embryos (blue) and mRNA-seq

759 (gray) in reads per million. Inset shows a magnification of the Aqz C-terminal region where

760 protein extension may occur.

761

762 Figure 3. $a q z^{G F P}$ mutant phenotypes. (A) and (B) show mutant ( $\left.a q z^{G F P} / a q z^{G F P}\right)$ and wild type

763 cuticles, respectively, the former one with an anterior hole and head involution defects (white 764 arrow). (C) and (E) show mutant embryos stained with neuronal and epithelial markers: in (C) 765 anti-Elav for neuronal bodies and anti-Coracle (Cora) for epithelial cells, while in (E) $22 \mathrm{C} 10$ for axons and some neuronal bodies. (D) and (F) show the same stainings using wild type control embryos. Scale bar is $100 \mu \mathrm{m}$. (G) Shows the percentages of embryos with neural phenotypes in $a q z^{G F P} / a q z^{G F P}$ embryos, with one or two copies of a genomic rescue construct. One copy of the rescue construct rescues significantly the mutant phenotype, whereas two copies effects a 
770 significant, but smaller, rescue, pointing to some gain-of-function effects with two rescue

771 constructs. Two copies of the rescue construct in a wild type background have neurogenic

772 phenotypes in over $40 \%$ of embryos, phenotypes similar to aqz mutants. In $(\mathrm{G})$ and $(\mathrm{H}) a q z^{G F P}$ is

773 homozygous mutant, $a q z^{R E C U E /+}$ is one copy of the rescue construct, and $a q z^{r e c u e}$ indicates two

774 copies of the rescue construct. (H) Extended phenocritical period of $a q z^{G F P} / a q z^{G F P}$ mutants. The

775 surviving $a q z^{G F P} / a q z^{G F P}$ mutant embryos die majorly as larvae, with approximately $20 \%$

776 reaching pupariation. No adults are ever observed. The $a q z^{G F P} / a q z^{G F P}$ lethality is rescued to wild

777 type levels with one copy of the $a q z$ genomic rescue construct, but two copies of the rescue

778 construct, while having a significant rescue effect, are less effective, pointing to some gain-of-

779 function effects with two rescue constructs. (I) $a q z^{G F P} / a q z^{G F P}$ mutant pupae die early, with

780 necrosis (left side pupae, black arrow), compared to a control pupae (right side pupae). Scale bar

781 is $1 \mathrm{~mm}$. (J) Rescued $a q z^{G F P} / a q z^{G F P}$ mutant fly (top) compared to sibling control fly (bottom, has

782 curved wings as marker). In this and all figures, ** denotes significant differences. In all figures,

783 for all embryo experiments, representative ones are shown, and $\mathrm{n}$ examined is over 200 per 784 condition.

785

786

787

Figure 4. Aqz mRNAs and proteins are expressed throughout embryogenesis in the

788 ectoderm and in ectodermally derived structures, and have maternal contribution. (A) Anti-sense aqz in situ hybridization results at different embryonic stages of development; (B)

789 shows results with the sense control probe (both derived from $a q z$ cDNA LD47990). Embryos in (C) are stained with the anti-Aqz antibody, (1:100), whereas (D) shows expression of chimeric

791 GFP::Aqz proteins using an anti GFP antibody (1:100) from the same embryos, which are

792 heterozygous for $a q z^{G F P}$, and thus, have expression of both GFP::Aqz and native Aqz protein.

793 As shown in (E), results from both are largely coincidental except some PNS cells and some

794 amnioserosa cells, only labeled with the anti-Aqz antibody. In all panels, anterior is left and

795 dorsal is up. All views are lateral views except the lower row, where ventrolateral views are

796 shown to evidence the developing central nervous system. Stage 5 early syncytial embryo, stage

797 9-10 germband extension/retraction stages, stage 14 dorsal closure, and stage 17, end of

798 embryogenesis. Scale bar is $100 \mu \mathrm{m}$. 
800 Figure 5. Aqz expressed in ectodermally derived tissue. Panel (A) shows an anti-GFP staining

801 for GFP::Aqz expression together with an epidermal marker, anti-Cora, showing some

802 coincidental expression (yellow). For all immune-stainings in the figure, the panels marked

803 underneath the top panels A-D labeled E-F show the GFP::Aqz channel separate, and the panels

804 G-H show the anti-Aqz. The bottom row of panels marked with I, J, K and L depict the anti-

805 coracle (I), anti-Elav (J), anti-22C10 (K), and anti-repo (L) channels, respectively. Panel (B)

806 shows anti-GFP for GFP::Aqz and anti-Elav, to mark neuronal cells in the developing central

807 NS. (C) Using monoclonal 22C10, there is coincidental expression with anti-Aqz antibody,

808 showing that some neurons in the PNS are positive for both Aqz and 22C10 (yellow). (D) In

809 contrast no glial cells (marked by anti-Repo antibody) are coincidental with anti-Aqz antibody

810 staining, showing that glial cells do not express Aqz. Scale bar is $100 \mu \mathrm{m}$.

811

812 Figure 6. $a q z$ NS defects are detected from the onset of NS formation. (A, C, E and G) are $a q z$

813 homozygous mutant embryos stained with markers for the NS. (B, D, F and H) are 814 corresponding heterozygous embryos (controls). All views are lateral with anterior to the left,

815 dorsal up. A and B show anti-Achaete staining marking proneural cell during germband 816 extension/retration. $\mathrm{C}$ and $\mathrm{D}$ show deadpan marked neuroblasts during germband 817 extension/retraction and condensation of the NS. Notice irregular appearance of neuroblasts in C 818 compared with D (control). E and F show expression of Evenskipped (Eve), marking ganglion 819 mother cells, showing a disruption in the mutant (compare E to F) at this later stage of 820 development (stage 15). G and H mark expression of GFP::Aqz. Green lines underneath G mark 821 parts of the embryo with abnormal GFP::Aqz expression, compared to H, where the GFP::Aqz 822 signal is distributed homogeneously. I marks neuroblasts. They also show a clumped and 823 irregular distribution (compare to $\mathrm{J}$ which shows a regular array of neuroblasts). Red lines mark 824 areas with abundant neuroblasts in I. K and L show the merged images. Scale bar is $100 \mu \mathrm{m}$.

825

826 Figure 7. Mutations in Aqz lead to irregular distribution of epithelial tissue and NS. A and 827 O show lateral views of embryos (anterior to the left and dorsal up) stained for neuroblasts 828 (Deadpan antibodies, red), epithelia (Coracle antibodies, yellow), also showing expression of 829 Aqz (green). Scale bar is $100 \mu \mathrm{m}$. In A, two distinct regions showing irregular distribution of 830 labels: A1 and A2, are enclosed in white rectangles and enlarged to the right. For comparison, a 
831 similar control area is shown in $\mathrm{O}$, the heterozygous control embryo. B', F and P show enlarged

832 anti-Deadpan staining (red); C, G and Q show enlarged GFP::Aqz expression, D, H and R show

833 enlarged anti-Coracle expression, whilst E, I and S show merged images, respectively. Scale bar

834 is $50 \mu \mathrm{m}$. J-N show another example of lack of epithelial cells using a different epidermal marker

835 (anti-FasIII, K) and an irregular distribution of neuroblasts (anti-Deadpan, L) in $a q z^{G F P}$ mutant

836 embryos (GFP::Aqz, J). Nuclei are labeled by DAPI (M). N show the respective merged image.

837

838 Figure 8. The $a q z$ mutant neuroepthelium has polarity defects. A, E, I, M, Q, and U are lateral 839 views of embryos undergoing dorsal closure, with anterior to the left and dorsal up. Bars are 100

$840 \mu \mathrm{m}$. A-D, I-L, and Q-T are $a q z^{G F P}$ mutant embryos, whereas E-H, M-P, and U-X are $a q z^{G F P}$

841 heterozygtes as controls. B, F, J, N, R, and V show GFP::Aqz expression, C and G anti-Crumbs

842 (Crb) (red), $\mathrm{K}$ and $\mathrm{O}$ anti-Discs large (Dlg) (red), and S and W anti-Delta (Dl) (yellow). D, H,

$843 \mathrm{~L}$, and $\mathrm{P}$ show respective merged images. Scale bar is $50 \mu \mathrm{m}$. T and X show anti-DE-cadherin

844 (DE-Cad) (red). Note that homozygous mutant GFP::Aqz expression appears more clumped

845 compared to the heterozygous controls, as stated before, and aids in identification of mutant

846 embryos.

847

848 Figure S1. aqz locus cDNAs partially sequenced. We sequenced both the 5' and 3' ends of 849 SD19655 and RE74095 to help us piece together the extent of the transcripts of the locus. 850

851 Figure S2. Other $a q z$ mutant alleles have ectoderm-derived mutant phenotypes. (A) shows 852 cuticular phenotypes; mutant embryos have holes in various positions (dorsal, lateral, anterior), 853 compared to a wild type cuticle (bottom left). (B) shows nervous system staining using the Elav 854 staining, where clear deformities of the central nervous system are shown, compared to the wild 855 type (bottom panel). (C) shows anti-22C10 staining marking neuronal somas and the peripheral 856 nervous system. aqz mutant embryos have irregularities in the disposition of the peripheral 857 nervous system, as well as abnormal regions of low and high density of peripheral nervous 858 system cells, compared to wild type (bottom panel). (D) shows anti-BP102 staining showing 859 abnormalities in $a q z$ mutant connectives in the ventral nervous chord in late embryos (wild type 860 control, bottom panel). All embryos are shown with anterior to the left and dorsal up. 
862 Figure S3. $a q z^{1}$ mutant phenotypes. (A) $a q z^{1}$ mutants have a cuticular phenotype, similar to $863 a q z^{G F P}$, but at a lower penetrance. $a q z^{1}$ cuticles have holes (arrows), compared to (B), a wild type

864 cuticle. Mutant $a q z^{1}$ embryos have nervous system phenotypes similar to $a q z^{G F P}$ as (C) shows a 865 disorganized and deformed nervous system stained with anti-Elav (top panel), and also shows 866 abnormal epithelial tissue, as marked by anti-Coracle staining (middle panel). Lower panel 867 shows a merged image. (D) shows a similarly stained control embryo. (E) shows an $a q z^{1}$ mutant 868 embryo displaying abnormal peripheral nervous system, as evidenced by anti-22C10 staining, 869 compared to (F), a wild type control embryo. In all panels, embryos are shown with dorsal up 870 and anterior left. Scale bar are $100 \mu \mathrm{m}$. (F) shows a quantification of $a q z^{1}$ nervous system 871 phenotypes, akin to neurogenic phenotypes, in roughly a third of mutant embryos. Two copies of 872 a genomic rescue construct significantly increase this percentage: over $60 \%$ embryos with the 873 rescue constructs exhibit a neurogenic phenotype. (H) The majority of $a q z^{1}$ embryos that survive 874 embryogenesis die as pupae, with a few (less than 10\%) dying as larvae. Adding two aqz 875 genomic rescue constructs significantly increases the number of larvae (over 50\%) that do not 876 reach pupation. In $(\mathrm{G})$ and $(\mathrm{H})$ adding wild type copies of $a q z$ increases the $a q z^{1}$ mutant 877 phenotypes, similar to the effect of two rescue wild type copies of $a q z$ in $a q z^{G F P}$ homozygotes.

878 (I) shows two $a q z^{1}$ mutant pupae with necrotic patches (black arrows) (left and middle pupae), compared to a wild type control pupa (right). Scale bar $1 \mathrm{~mm}$. Anterior is up.

880

881 Figure S4. $a q z^{1}$ has mutant phenotypes in epidermis and nervous system. A and C show mutant 882 embryos, while B and D show similarly stained control embryos (heterozygotes). A and B show 883 anti-Deadpan for neuroblasts, and anti-Fascilin III to mark the epithelium. Note disorganization 884 in the mutant embryos. Scale bar is $100 \mu \mathrm{m}$. C and D show anti-BP102 staining, showing 885 abnormalities in mutant connectives in the ventral nervous chord. All embryos are oriented 886 dorsal up and anterior left.

887

888 Table 1. $a q z$ alleles complementation matrix. $a q z^{i n s e r t i o n}\left(a q z^{2}\right)$ is homozygous viable and 889 complements $a q z^{1}$, which is homozygous lethal. $a q z^{G F P}$ (another P-element insertion), is 890 homozygous lethal. $a q z^{1}$ complements $a q z^{G F P}$ and all three $a q z$-uncovering deficiencies 891 (Df(3R)Exel6150, $D f(3 R) B S C 478$, and $D f(3 R) B S C 506)$. aqz ${ }^{G F P}$ does not complement these 892 deficiencies. + Indicates complementation, L indicates non-complementation (lethality), HV 
893 indicates homozygous viable, and HL indicates homozygous lethal. $D f(3 R)$ Exel6150 deletes 894 85A5-B6, $D f(3 R) B S C 478$ deletes 85A5-B8, and $D f(3 R) B S C 506$ deletes 85B1-C2. Together, the 895 common deleted fragment goes from 85B1-B6. The aqz locus is located at $85 \mathrm{~B} 2$.

896

897 Table S1. $D f(3 R)$ Exel6150 and $a q z^{G F P}$ genetically complement stocks with insertions on $a q z$ 898 neighboring genes. $D f(3 R)$ Exel6150 and $a q z^{G F P}$ are homozygous lethal, but fully complement 899 insertions in CG9836, CR43130, CG9837 and CG8359. + indicates complementation and ND 900 indicates not determined. The crosses between $D f(3 R)$ Exel6150 and mutant alleles 901 produced adults with and without balancers (for $P\{E P\} I s C U G 18758$ (CG9836) $=70$ with and 90241 without balancers, for $P\{S U P o r-P\} K G 08159$ ry $506(C R 43130)=18$ with and 82 without 903 balancers and for $M i\{M I C\} C G 9837 M I 01411(C G 9837)=49$ with and 81 without balancers). Also 904 the crosses between $a q z^{G F P}$ and mutant alleles produced adults with and without balancers 905 (for P\{EP\}IscUG18758(CG9836) $=105$ with and 77 without balancers, for P\{SUPor$906 P\} K G 08159$ ry506(CR43130) $=106$ with and 64 without balancers, for $907 \mathrm{Mi}\{M I C\} C G 9837 M I 01411(C G 9837)=35$ with and 53 without balancers and $908 P\{E P g y 2\} h n g 2 E Y 18943(C G 8359)=23$ with and 50 without balancers). 


\section{Table $\mathbf{1}$ (on next page)}

aqz alleles complementation matrix.

$\operatorname{aqz} z^{\text {insertion }}\left(a q z^{2}\right)$ is homozygous viable and complements $a q z^{1}$, which is homozygous lethal. $a q z^{G F P}$ (another P-element insertion), is homozygous lethal. $a q z^{1}$ complements $a q z^{G F P}$ and all three aqz-uncovering deficiencies (Df(3R)Exel6150, Df(3R)BSC478, and Df(3R)BSC506). $a q z^{G F P}$ does not complement these deficiencies. + Indicates complementation, $L$ indicates non-complementation (lethality), HV indicates homozygous viable, and HL indicates homozygous lethal. $D f(3 R)$ Exel6150 deletes 85A5-B6, Df(3R)BSC478 deletes 85A5-B8, and $D f(3 R) B S C 506$ deletes $85 B 1-C 2$. Together, the common deleted fragment goes from 85B1B6. The aqz locus is located at $85 \mathrm{~B} 2$. 
1 Table 1

\begin{tabular}{|c|c|c|c|c|c|c|}
\hline Allele & $a q z^{1}$ & $a q z^{2}$ & $a q z^{G F P}$ & $\begin{array}{c}\mathrm{Df}(3 \mathrm{R}) \text { Exel61 } \\
50\end{array}$ & $\begin{array}{c}\mathrm{Df}(3 \mathrm{R}) \mathrm{BSC} 4 \\
78\end{array}$ & $\begin{array}{c}\mathrm{Df}(3 \mathrm{R}) \mathrm{BSC} 50 \\
6\end{array}$ \\
\hline$a q z^{1}$ & $\mathrm{HL}$ & + & + & + & + & + \\
\hline$a q z^{2}$ & & $\mathrm{HV}$ & + & + & + & + \\
\hline$a q z^{G F P}$ & & & $\mathrm{HL}$ & $\mathrm{L}$ & $\mathrm{L}$ & $\mathrm{L}$ \\
\hline $\begin{array}{c}\operatorname{Df}(3 \mathrm{R}) \operatorname{Exel} 615 \\
0\end{array}$ & & & & HL & $\mathrm{L}$ & $\mathrm{L}$ \\
\hline Df(3R)BSC478 & & & & & HL & $\mathrm{L}$ \\
\hline Df(3R)BSC506 & & & & & & $\mathrm{HL}$ \\
\hline
\end{tabular}

2

3

4

5 


\section{Figure 1}

The aaquetzalli (aqz) locus codes for at least three alternatively spliced transcripts

(A) aqz encompasses a little above $7 \mathrm{~Kb}$, from 4647000 to 4638000 at the base of chromosome $3 \mathrm{R}$ at $85 \mathrm{~B} 2$; in the figure the genes are oriented such that the $5^{\prime}$ end of aqz is to the left. Besides aqz (solid black arrow), other genes mapping in the region are shown as grey-hashed arrows. aqz codes for four predicted different mRNA species, each with two exons (grey boxes) and one intron (black line). Several cDNAs and ESTs (white boxes with black borders, and white boxes with black dotted lines, respectively) map in the locus. Underneath the depicted chromosome line a grey bar signals the extent of the genomic rescue construct employed (BAC CH322-25015), inserted in the second chromosome at $53 \mathrm{~B}$ 2 (Bloomington line \#9736). The location of two transposon insertion mutants, one in the promoter region $\left(a q z^{2}\right)$, and one in the intron $\left(a q z^{G F P}\right)$, are shown. $a q z^{G F P}$ is a fly trap line (\#CB03335), coding for a predicted fusion protein, where the GFP moiety is fused to the 5' end of Aqz. aqz ${ }^{1}$ has a four base pairs insertion, depicted in bold, in the promoter region of aqz between the predicted TATA box and initiation of transcription. aqz ${ }^{1}$ was isolated as an imprecise excision of the $a q z^{2}$ transposon. Nearly all isolated aqz alleles are let $h$ al. (B) Aqz has a conserved domain at the 5' end of the protein. The homology is for a proline-rich conserved domain of nuclear receptor co-activators (PNRC-domain proteins) in single letter amino acid code, present in both invertebrate and vertebrate proteins. Numbers to the left indicate position of the domain within the proteins. (C) Northern blot using two different aqz cDNAs as probes reveal three different transcripts of 7.53, 5.03 and $3.16 \mathrm{~Kb}$. The smallest band ( $3.16 \mathrm{~Kb}$ ) is only present in adult flies, and is only seen using a 5' c DNA (LD47990), present in all aqz isoforms. The same blot that was used with the first probe, was photographed, then stripped, and re-probed with the a more 3' CDNA (LD02060), that recognizes aqz RB, RC, and RD, but not aqz RA. $n>10$. (D) Aqz mutant and control Western blot using an antibody against GFP recognizes a band at approximately $40 \mathrm{kD}$ (right black 
arrow) in $a q z^{G F P}$ homozygotes, and weakly, in $a q z^{G F P}$ heterozygotes. All other weak bands are present in all lanes, including the control, wild type lane, and are, consequently, not specific.

A

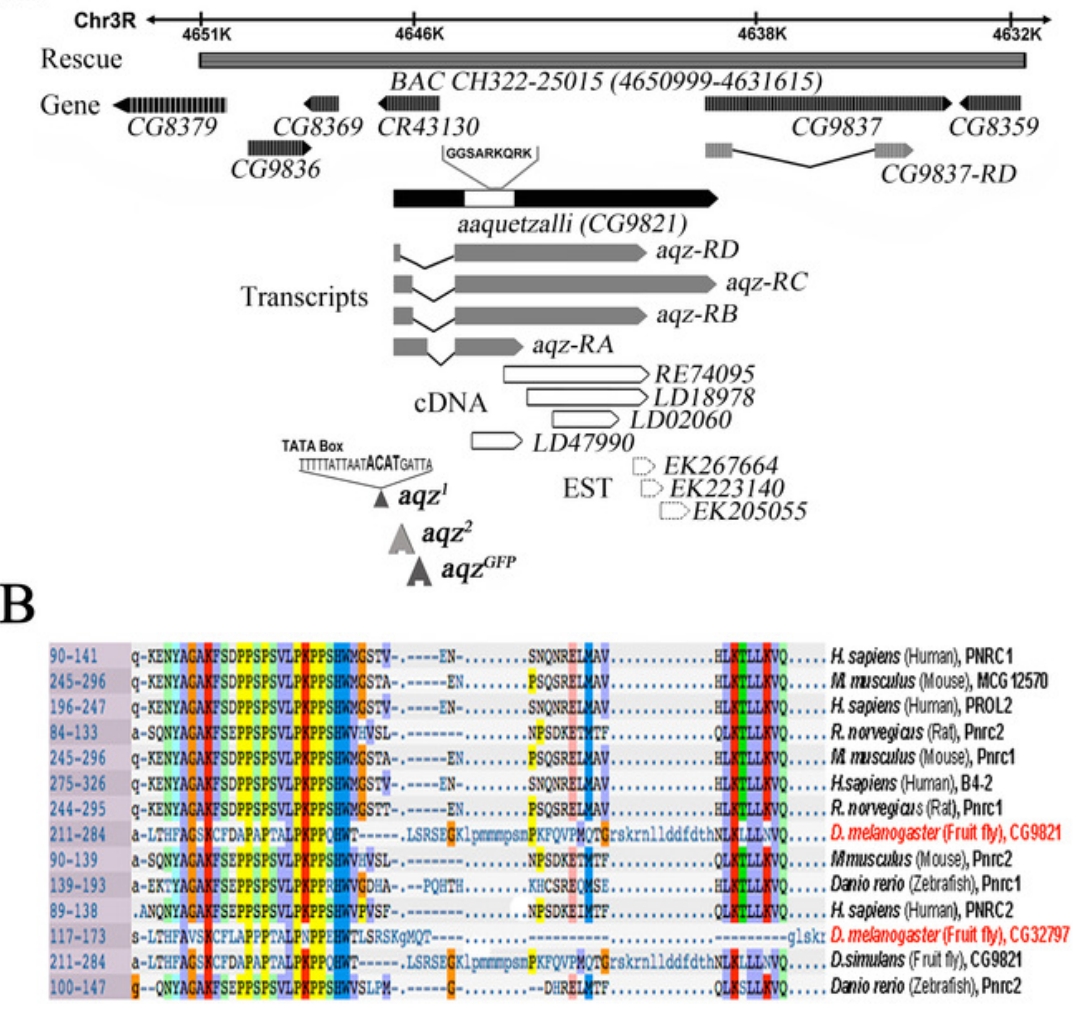

$\mathrm{C}$

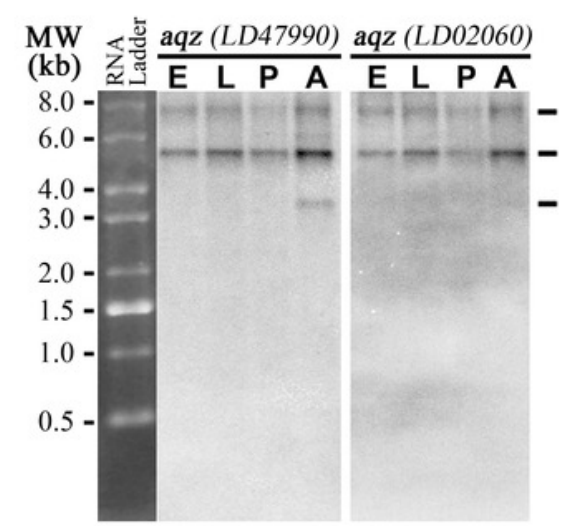

$\mathrm{D}$

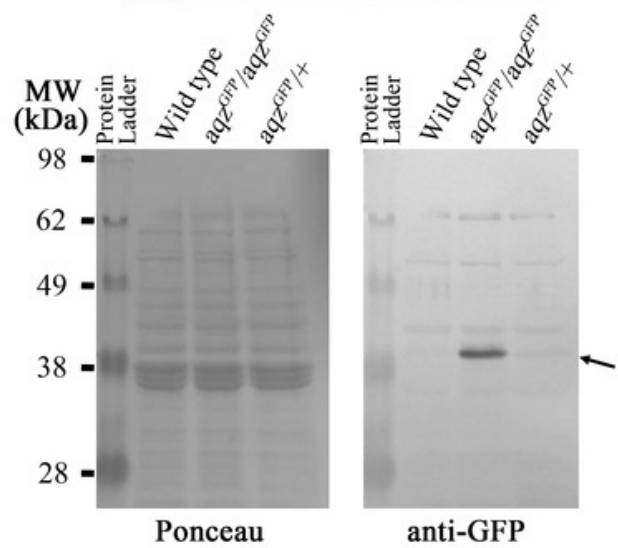




\section{Figure 2}

aqz has alterative splicing.

aqz mRNAs first exon is translated, and some have also 3' stop codon read-through in the second exon, judging from ribosome footprinting data. The aqz locus (CG9821) together with the annotated predicted mRNAs are depicted on top (green) with dotted lines depicting introns, and red lines depicting other transcripts. The neighboring CG9837 locus, overlapping CG9821 at the $5^{\prime}$ end, is also depicted in green. The ribosome footprinting data is depicted below taken from S2 cells (black), 0-2 hrs. embryos (blue) and mRNA-seq (gray) in reads per million. Inset shows a magnification of the Aqz C-terminal region where protein extension may occur. 


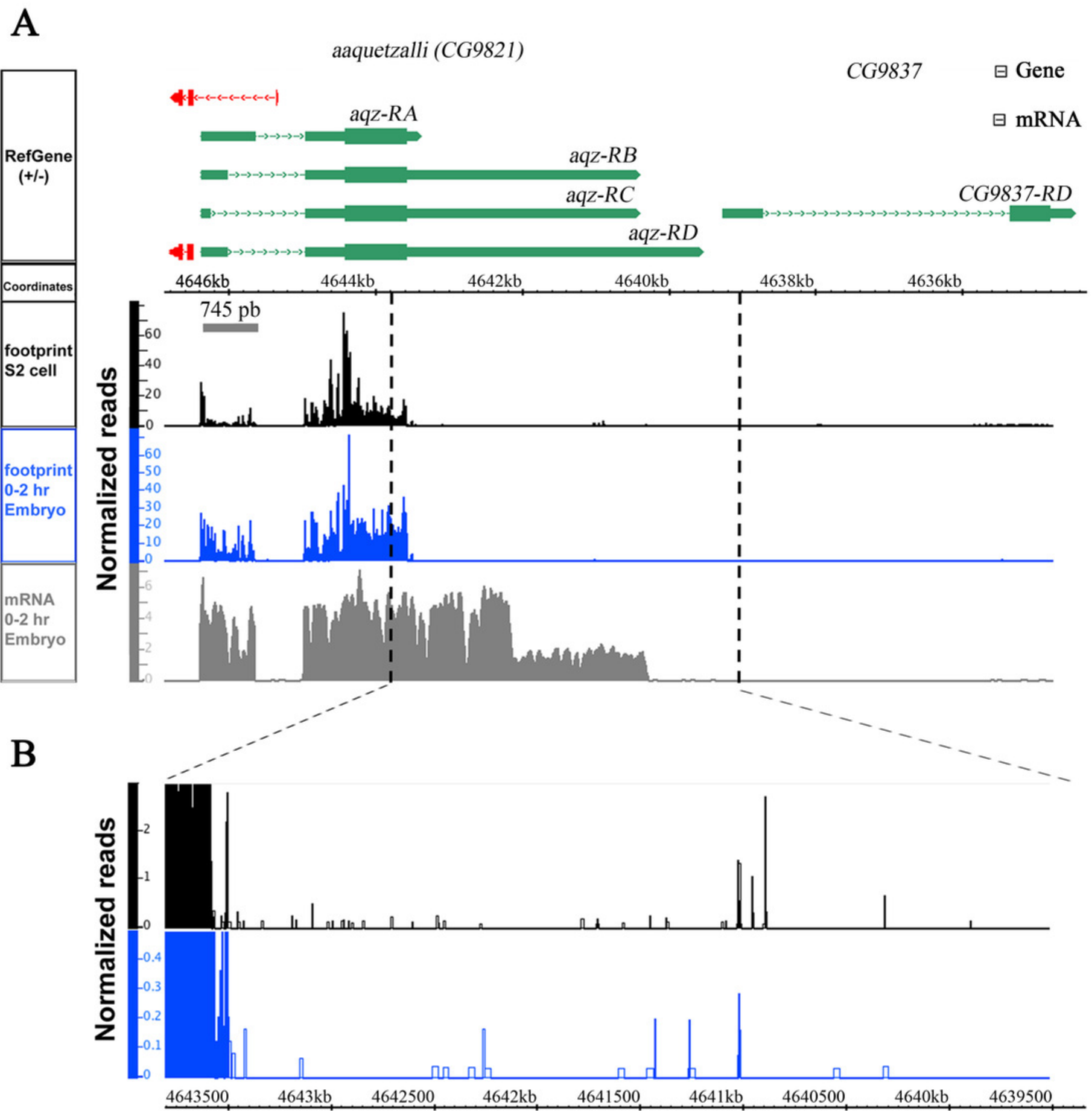




\section{Figure 3}

aqz ${ }^{G F P}$ mutant phenotypes

(A) and (B) show mutant (aqz $\left.z^{G P} / a q z^{G F P}\right)$ and wild type cuticles, respectively, the former one with an anterior hole and head involution defects (white arrow). (C) and (E) show mutant embryos stained with neuronal and epithelial markers: in (C) anti-Elav for neuronal bodies and anti-Coracle (Cora) for epithelial cells, while in (E) 22C10 for axons and some neuronal bodies. (D) and (F) show the same stainings using wild type control embryos. Scale bar is 100 $\mu \mathrm{m}$. (G) Shows the percentages of embryos with neural phenotypes in $a q z^{G P} / a q z^{G F P}$ embryos, with one or two copies of a genomic rescue construct. One copy of the rescue construct rescues significantly the mutant phenotype, whereas two copies effects a significant, but smaller, rescue, pointing to some gain-of-function effects with two rescue constructs. Two copies of the rescue construct in a wild type background have neurogenic phenotypes in over $40 \%$ of embryos, phenotypes similar to aqz mutants. In (G) and (H) aqz ${ }^{G F P}$ is homozygous mutant, aqz ${ }^{\text {RECUE/ }}$ is one copy of the rescue construct, and aqz ${ }^{\text {recue }}$ indicates two copies of the rescue construct. (H) Extended phenocritical period of aqz ${ }^{G P P} / a q z^{G P P}$ mutants. The surviving $a q z^{G F P} / a q z^{G F P}$ mutant embryos die majorly as larvae, with approximately $20 \%$ reaching pupariation. No adults are ever observed. The $a q z^{G F P} / a q z^{G F P}$ lethality is rescued to wild type levels with one copy of the aqz genomic rescue construct, but two copies of the rescue construct, while having a significant rescue effect, are less effective, pointing to some gainof-function effects with two rescue constructs. (I) $a q z^{G F P} / a q z^{G F P}$ mutant pupae die early, with necrosis (left side pupae, black arrow), compared to a control pupae (right side pupae). Scale bar is $1 \mathrm{~mm}$. (J) Rescued aqz ${ }^{G F P} / a q z^{G F P}$ mutant fly (top) compared to sibling control fly (bottom, has curved wings as marker). In this and all figures, ** denotes significant differences. In all figures, for all embryo experiments, representative ones are shown, and $\mathrm{n}$ examined is over 200 per condition. 

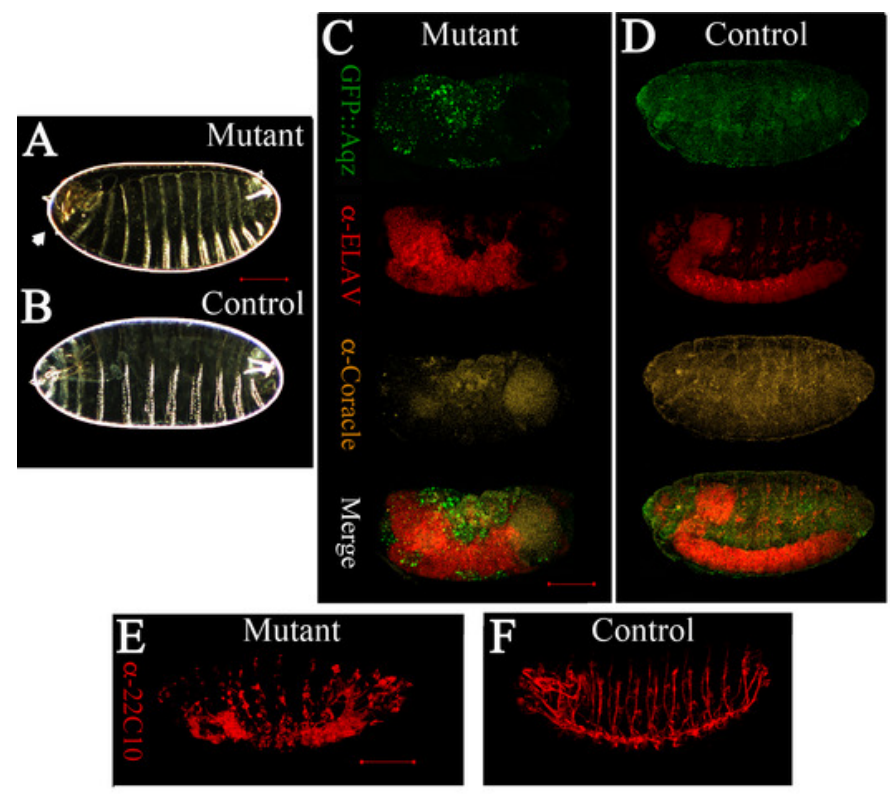

I

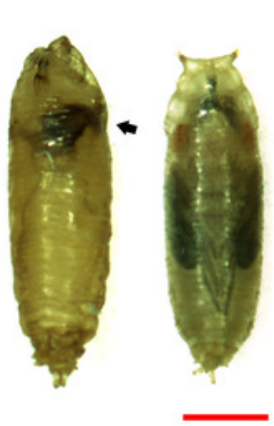

G

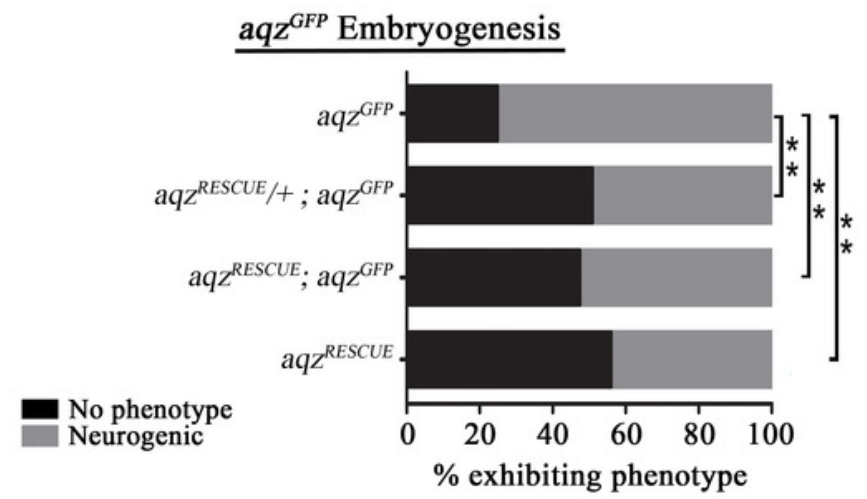

$\mathrm{H}$

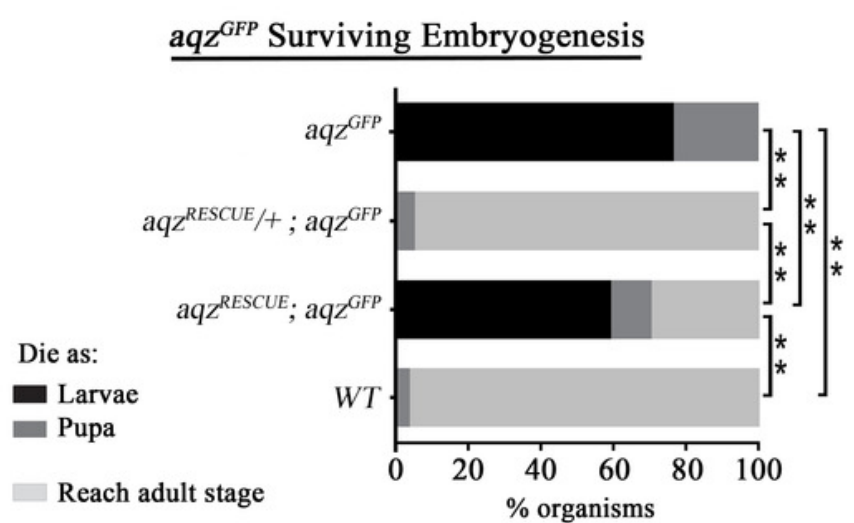




\section{Figure 4}

Aqz mRNAs and proteins are expressed throughout embryogenesis in the ectoderm and in ectodermally derived structures, and have maternal contribution.

(A) Anti-sense aqz in situ hybridization results at different embryonic stages of development;

(B) shows results with the sense control probe (both derived from aqz CDNA LD47990).

Embryos in (C) are stained with the anti-Aqz antibody, (1:100), whereas (D) shows expression of chimeric GFP::Aqz proteins using an anti GFP antibody (1:100) from the same embryos, which are heterozygous for aqz ${ }^{G P P}$, and thus, have expression of both GFP::Aqz and native Aqz protein. As shown in (E), results from both are largely coincidental except some PNS cells and some amnioserosa cells, only labeled with the anti-Aqz antibody. In all panels, anterior is left and dorsal is up. All views are lateral views except the lower row, where ventrolateral views are shown to evidence the developing central nervous system. Stage 5 early syncytial embryo, stage 9-10 germband extension/retraction stages, stage 14 dorsal closure, and stage 17, end of embryogenesis. Scale bar is $100 \mu \mathrm{m}$.

*Note: Auto Gamma Correction was used for the image. This only affects the reviewing manuscript. See original source image if needed for review.

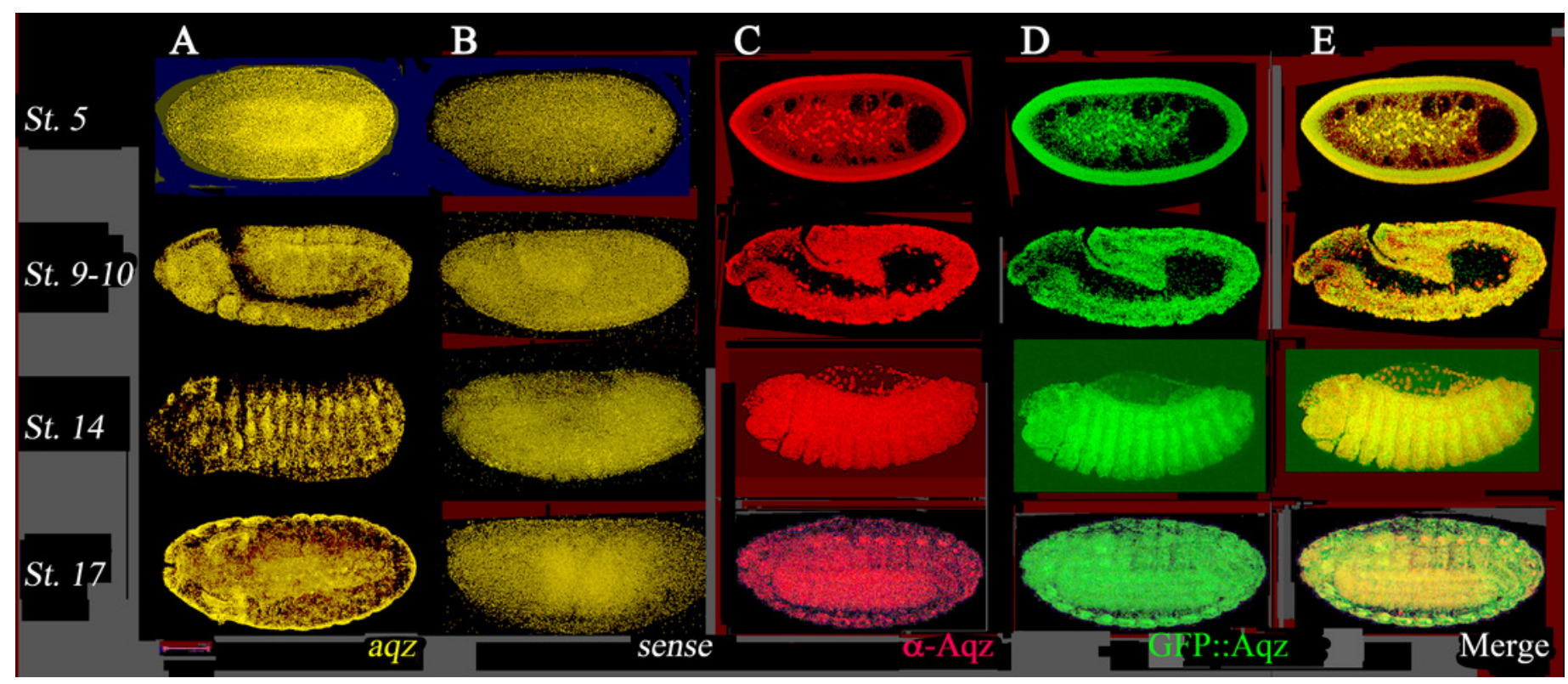




\section{Figure 5}

Aqz expressed in ectodermally derived tissue.

Aqz expressed in ectodermally derived tissue.Panel (A) shows an anti-GFP staining for GFP::Aqz expression together with an epidermal marker, anti-Cora, showing some coincidental expression (yellow). For all immune-stainings in the figure, the panels marked underneath the top panels A-D labeled E-F show the GFP::Aqz channel separate, and the panels G-H show the anti-Aqz. The bottom row of panels marked with I, J, K and L depict the anti-coracle (I), anti-Elav (J), anti-22C10 (K), and anti-repo (L) channels, respectively. Panel (B) shows anti-GFP for GFP::Aqz and anti-Elav, to mark neuronal cells in the developing central NS. (C) Using monoclonal 22C10, there is coincidental expression with anti-Aqz antibody, showing that some neurons in the PNS are positive for both Aqz and 22C10 (yellow). (D) In contrast no glial cells (marked by anti-Repo antibody) are coincidental with anti-Aqz antibody staining, showing that glial cells do not express Aqz. Scale bar is $100 \mu \mathrm{m}$.
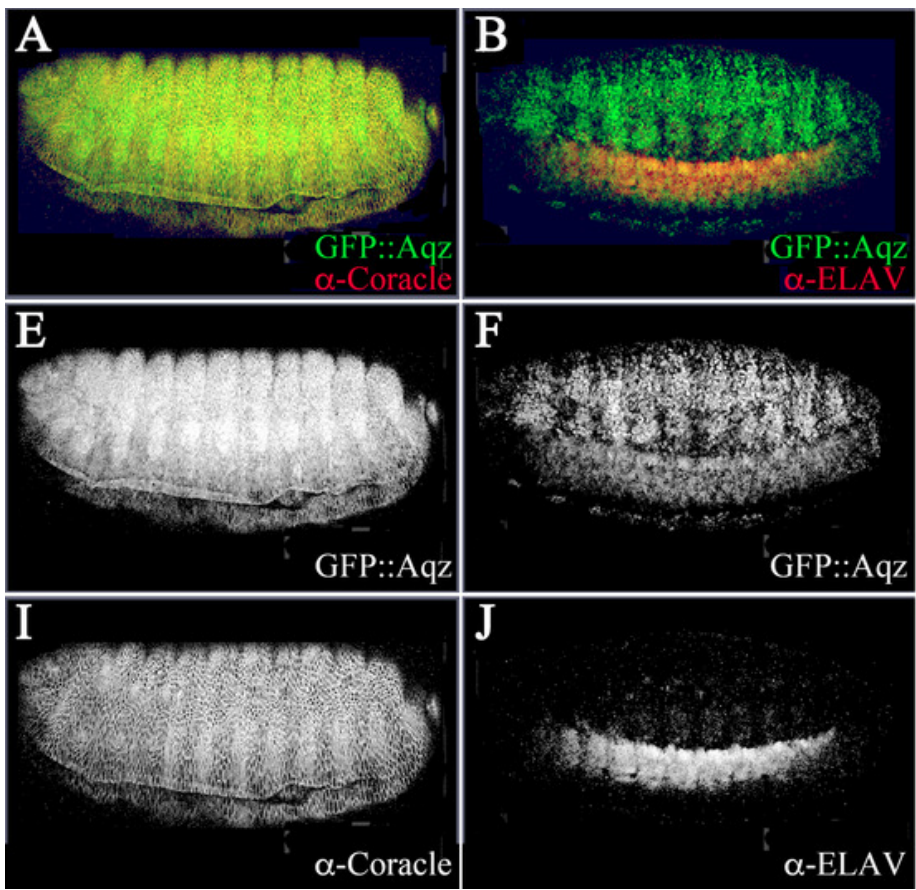
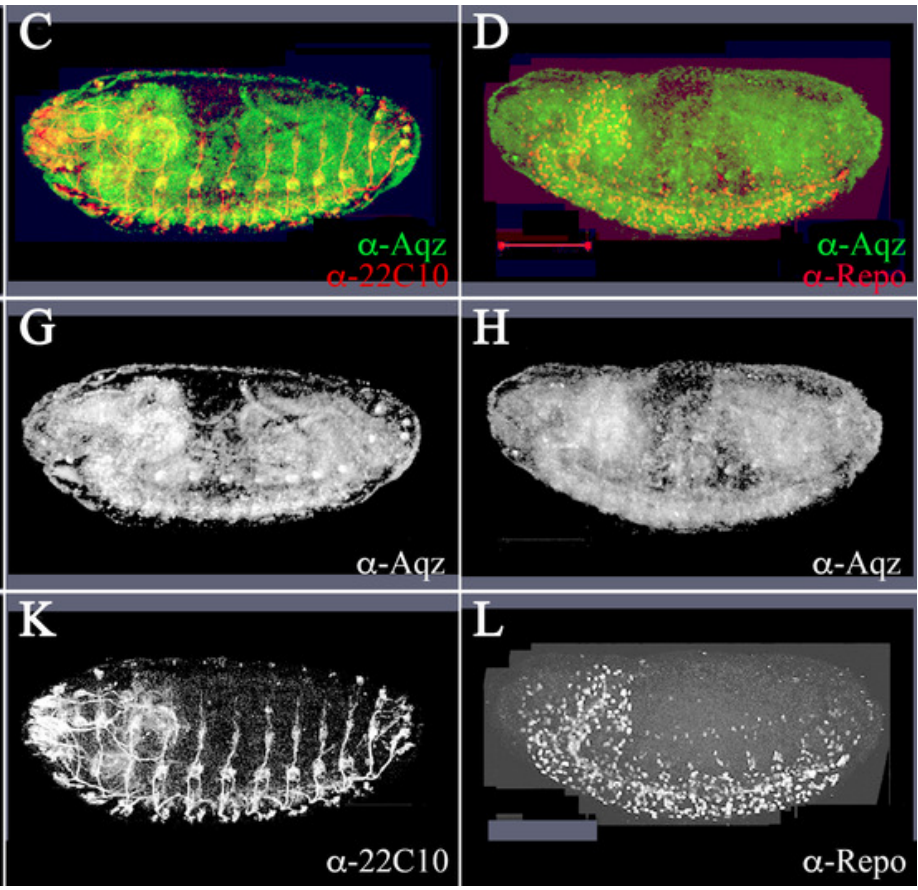


\section{Figure 6}

aqzNS defects are detected from the onset of NS formation.

aqzNS defects are detected from the onset of NS formation. (A, $C, E$ and $G$ ) are aqzhomozygousmutant embryos stained with markers for the NS. (B, D, F and H) are corresponding heterozygous embryos (controls). All views are lateral with anterior to the left, dorsal up. $A$ and $B$ show anti-Achaete staining marking proneural cell during germband extension/retration. $\mathrm{C}$ and $\mathrm{D}$ show deadpan marked neuroblasts during germband extension/retraction and condensation of the NS. Notice irregular appearance of neuroblasts in C compared with $D$ (control). E and F show expression of Evenskipped (Eve), marking ganglion mother cells, showing a disruption in the mutant (compare $E$ to $F$ ) at this later stage of development (stage 15). G and H mark expression of GFP::Aqz. Green lines underneath G mark parts of the embryo with abnormal GFP::Aqz expression, compared to $\mathrm{H}$, where the GFP::Aqz signal is distributed homogeneously. I marks neuroblasts. They also show a clumped and irregular distribution (compare to J which shows a regular array of neuroblasts). Red lines mark areas with abundant neuroblasts in $\mathrm{I} . \mathrm{K}$ and $\mathrm{L}$ show the merged images. Scale bar is $100 \mu \mathrm{m}$. 


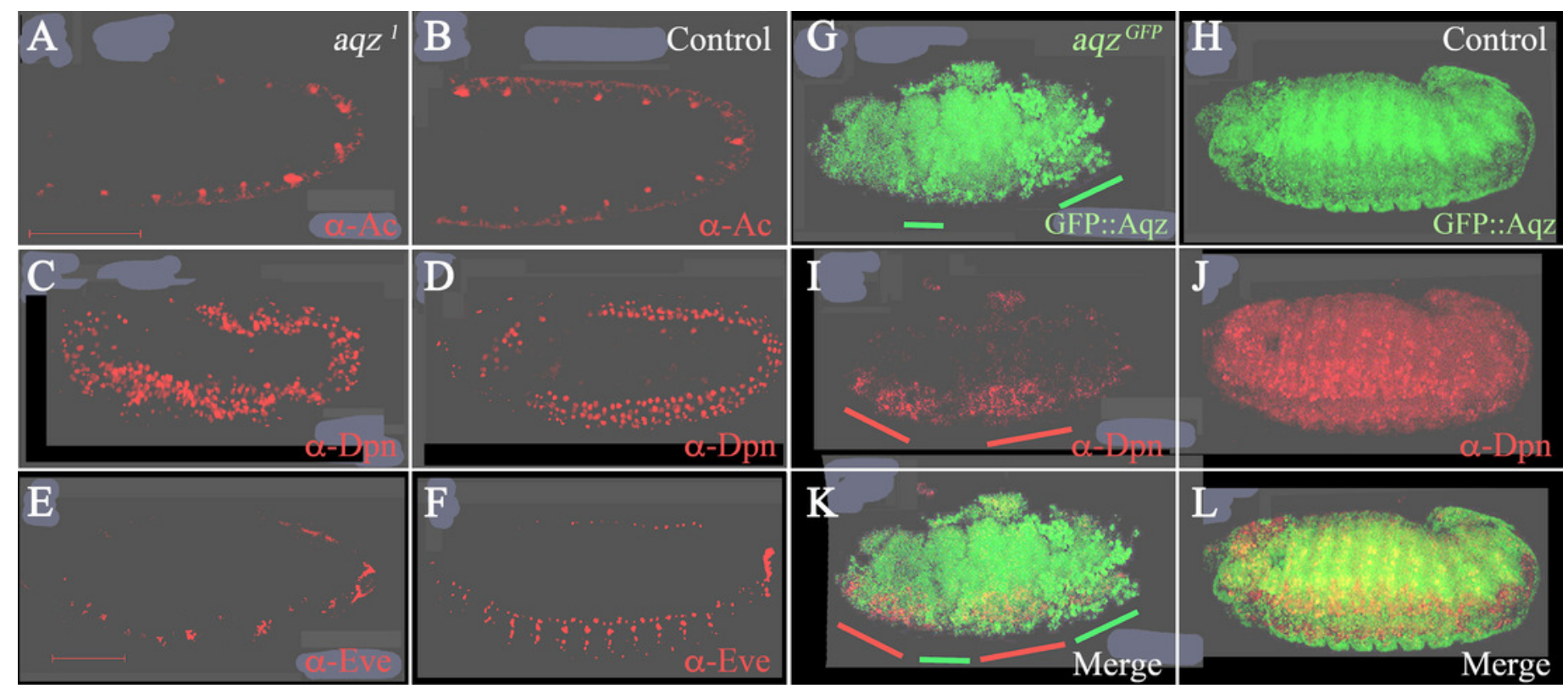




\section{Figure 7}

Mutations in Aqz lead to irregular distribution of epithelial tissue and NS.

Mutations in Aqz lead to irregular distribution of epithelial tissue and NS.A and $O$ show lateral views of embryos (anterior to the left and dorsal up) stained for neuroblasts (Deadpan antibodies, red), epithelia (Coracle antibodies, yellow), also showing expression of Aqz (green). Scale bar is $100 \mu \mathrm{m}$. In A, two distinct regions showing irregular distribution of labels: $\mathrm{A} 1$ and $\mathrm{A} 2$, are enclosed in white rectangles and enlarged to the right. For comparison, a similar control area is shown in $\mathrm{O}$, the heterozygous control embryo. $\mathrm{B}^{\prime}, \mathrm{F}$ and P show enlarged anti-Deadpan staining (red); C, G and Q show enlarged GFP::Aqz expression, $D, H$ and $R$ show enlarged anti-Coracle expression, whilst $E, I$ and $S$ show merged images, respectively. Scale bar is $50 \mu \mathrm{m}$.J-N show another example of lack of epithelial cells using a different epidermal marker (anti-FasIII, K) and an irregular distribution of neuroblasts (antiDeadpan, L) in aqz ${ }^{G F P}$ mutant embryos (GFP::Aqz, J). Nuclei are labeled by DAPI (M). N show the respective merged image.
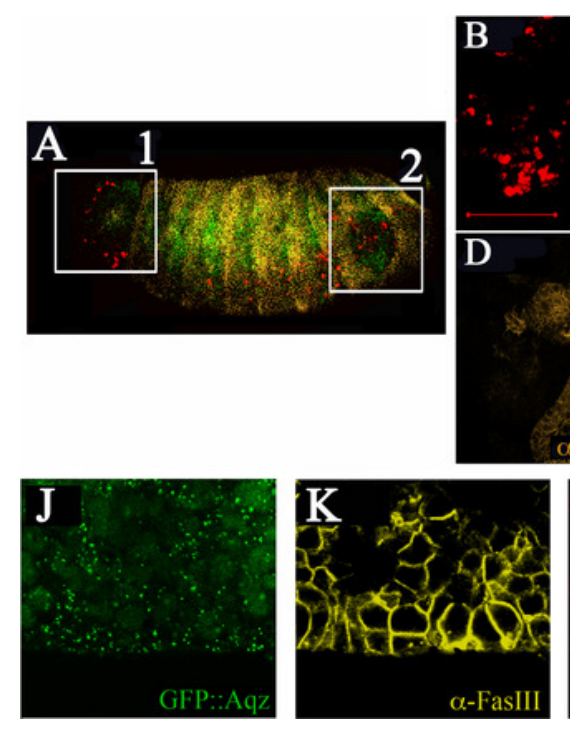
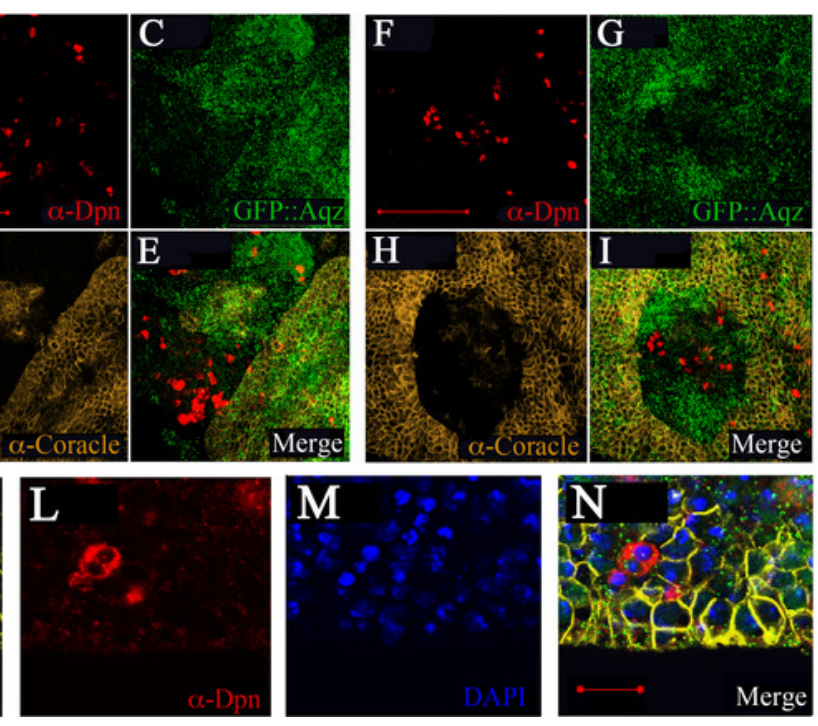
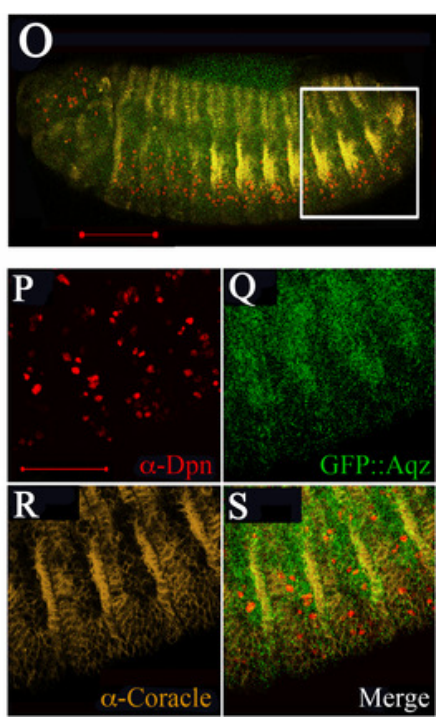


\section{Figure 8}

Theaqz mutant neuroepthelium has polarity defects.

Theaqz mutant neuroepthelium has polarity defects. $A, E, I, M, Q$, and $U$ are lateral views of embryos undergoing dorsal closure, with anterior to the left and dorsal up. Bars are $100 \mu \mathrm{m}$. A-D, I-L, and Q-T are aqz ${ }^{\mathrm{GFP}}$ mutant embryos, whereas E-H, M-P, and U-X are aqz ${ }^{G F P}$ heterozygtes as controls. B, F, J, N, R, and V show GFP::Aqz expression, C and $\mathrm{G}$ antiCrumbs (Crb) (red), $\mathrm{K}$ and $\mathrm{O}$ anti-Discs large (Dlg) (red), and S and W anti-Delta (DI) (yellow). $D, H, L$, and $P$ show respective merged images. Scale bar is $50 \mu \mathrm{m}$. $T$ and $X$ show anti-DEcadherin (DE-Cad) (red). Note that homozygous mutant GFP::Aqz expression appears more clumped compared to the heterozygous controls, as stated before, and aids in identification of mutant embryos. 


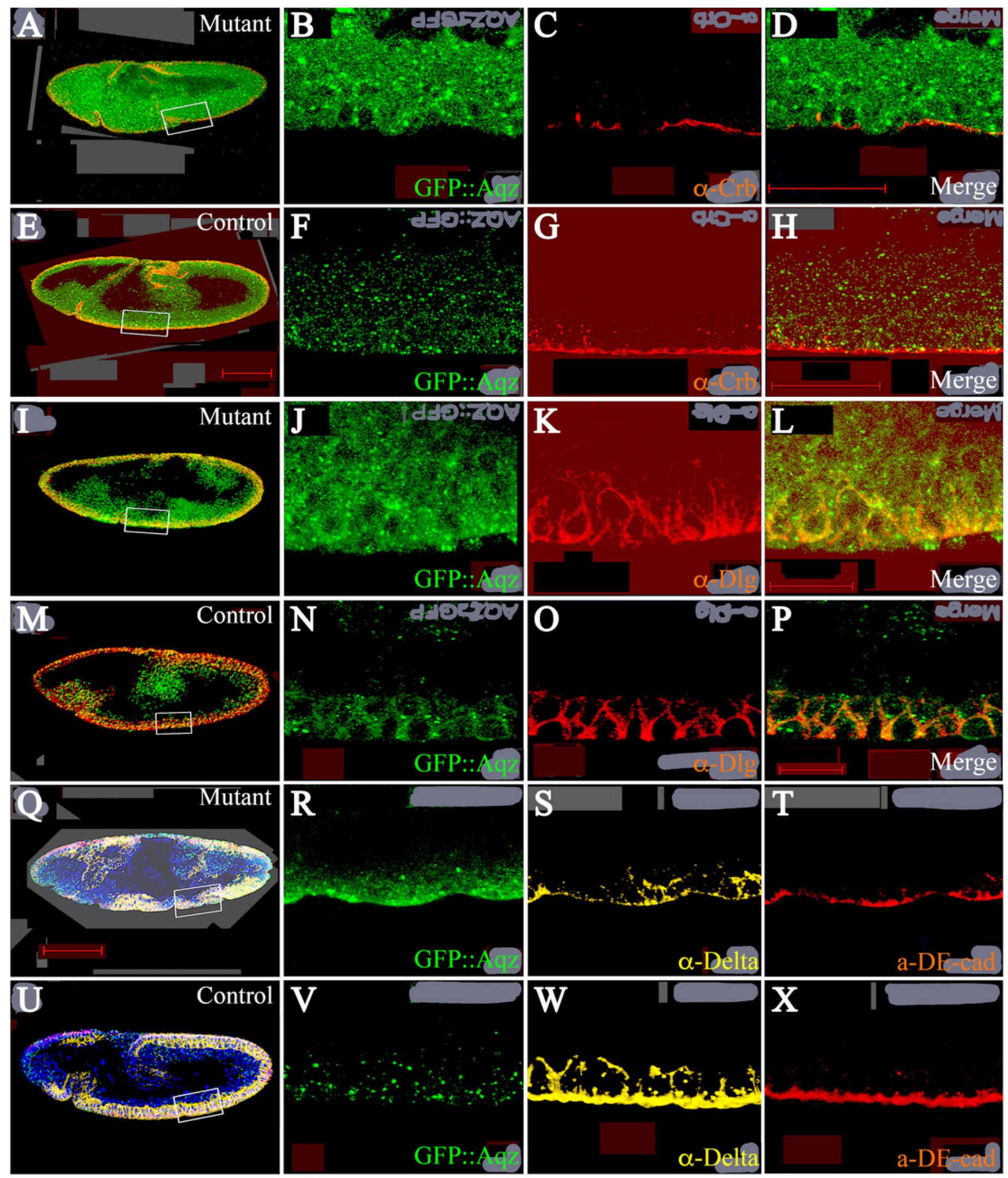

